\title{
Wave forces on marine structures with small speed in water of restricted depth
}

\author{
John Grue and Dag Biberg \\ Department of Mathematics, Mechanics Division \\ University of Oslo, Norway
}

Wave forces on three-dimensional bodies with small forward speed is considered. The water depth is allowed to be finite (or shallow), which is a generalization of existing theories. The problem is formulated consistently to leading order in the wave amplitude and the forward speed by means of integral equations. Linear forces and moments, second order horizontal drift force, vertical moment, and wave drift damping is considered. Results are presented for a half-immersed sphere, a vertical truncated cylinder and a ship. Considerable enhancement of the wave forces may occur when the wave number multiplied by the water depth is smaller than unity and the body draught is comparable to the water depth. The wave drift damping for a ship may then be up to three times larger than in deep water.

\section{Introduction}

Wave forces on floating bodies may be considerably influenced by a forward speed of the body or the presence of a current in the ocean. Especially this is true for the wave drift forces, which for large-volume bodies may be changed by $100 \%$ when the forward speed of the body or the current speed in the ocean increases from zero to $1-2 \mathrm{~ms}^{-1}$. The effect of a forward speed of the body is mathematically equivalent to the presence of a uniform current in the ocean and is here considered as one problem.

The pronounced effect of a forward speed on the wave drift forces introduces an important damping force of resonant slow drift motions of moored bodies, where damping due to linear wave scattering is negligible and viscous forces may be small. This damping force, denoted by wave drift damping, was first pointed out by Wichers and Van Sluijs ${ }^{1}$. The effect of a small forward speed on the wave loads, and wave drift damping of floating bodies, has recently been studied by assuming that the water depth is infinite (Huismans ${ }^{2}$, Zhao et al. ${ }^{3}$, Zhao and Faltinsen ${ }^{4}$, Nossen et al. ${ }^{5}$, Grue ${ }^{6}$, Emmerhoff and Sclavounos ${ }^{7}, N_{\text {ewman }}{ }^{8}$ ).

The intention with the present paper is to generalize the previous theories to allow for a finite value of the water depth. This may be relevant for several marine operations, as 
pointed out e.g. by Pinkster and Huijsmans ${ }^{9}$. They report both theoretical and experimental results for the mean drift force on a ship at zero forward speed when the ratio between the water depth and the draught of the ship is as small as 1.2. Their results exhibit a pronounced peak in the mean drift force when the wave length is comparable to the ship length, which is not observed in deeper water. We shall find that the forward speed part of the forces may be even more enhanced due to a reduced water depth than the zero speed forces.

The fluid motions and forces are in deep water determined by the wave slope, the wave number multiplied by the body dimension and the Froude number. A finite water depth adds two new parameters to the problem, however. One parameter is the gap between the floating body and the sea bottom which may be characterized by the ratio between the water depth, $h$, and the body draught, $T$, i.e. $h / T$. The other parameter is the ratio between the wave length and the water depth which is characterized by $K h$ ( $K$ the wave number).

The ratio between the group velocity in finite and infinite water depth appears in the formulas for the scattered wave characteristics. This ratio is close to unity when $K h>1$ but becomes proportional to $K h$, practically speaking, when $K h<1$, which means that the effect of a limited water depth then becomes important. The ratio between the group velocity in finite and infinite water depth also appears in the generalized Haskind relations for the linear exciting forces, the mean horizontal drift force, the mean vertical drift moment and the mean energy flux, which are quantities obtained by means of far field analysis. A finite water depth is then expected to significantly influence the forces when $K h<1$ and $h / T$ is close to unity, but not otherwise. This is confirmed by the numerical examples.

We assume that potential theory can be applied and that viscous effects may be neglected. Furthermore we assume that the wave slope is limited so that nonlinear interaction is not taking place in the wave field.

The floating body is advancing with a small forward speed while responding to incoming monochromatic waves. All six degrees of freedom are accounted for in the linear responses. The wave heading is arbitrary. A set of boundary value problems are formulated and a Green function for the linear time harmonic problem is derived. The problem is solved consistently to leading order in the wave amplitude and the forward speed by means of integral equations. Linear forces and moments are considered. The added mass and damping coefficients obey the Timman-Newman relations, and the linear exciting forces and moments are found either by pressure integration or by means of generalized Haskind relations. This provides checks of the model, which are confirmed by the numerical results.

The mean horizontal drift force is expressed in terms of the linear momentum flux in the far field. The mean vertical drift moment is, correspondingly, obtained by the angular momentum flux. These formulas may have particular relevance to ship maneuvering in shallow water. The wave drift damping is one of the important quantities to predict. We find that the wave drift damping for a ship may be three times larger at a small water depth than in deep water. More specifically this occurs when the water depth is slightly larger than the ship draught and the wave length is comparable to the ship length.

In $\S 2$ the boundary value problems are formulated. In $\S 3$ the linear problem is solved. We consider in $\S 4$ the linear forces and in $\$ 5$ the drift forces. Convergence of the method 
and the energy check are discussed in respectively $\S 6$ and $\S 7$.

\section{The boundary value problems}

The problem is considered in the frame of reference translating with the steady forward speed $U$ of the body. A coordinate system $O-x y z$ is introduced with the $x$-and $y$-axes in the mean free surface, the $z$-axis vertical upwards, and with unit vectors $i, j, k$ along the $x, y, z$-directions, respectively. The fluid layer is assumed to be of constant depth, with the sea bottom located at $z=-h$. In this frame of reference there is an ambient current with speed $U$ which we assume is directed along the negative $x$-axis. The fluid is assumed to be homogenous and incompressible, and the motion irrotational. The fluid velocity may then be written

$$
\mathbf{v}=U \nabla \chi_{\bullet}+\nabla \Phi+\nabla \psi^{(2)}
$$

where $\chi_{s}, \Phi$ and $\psi^{(2)}$ are Laplacian velocity potentials. $\chi_{s}$ denotes the potential due to the current and the steady flow around the body, $\Phi$ the linear time harmonic potential due to the incoming, scattered and radiated waves, and $\psi^{(2)}$ a steady potential being of second order in the wave amplitude.

\subsection{The steady potential $\chi_{s}$}

The velocity potentials are subject to boundary conditions at the free surface, the mean position of the body, the sea bottom and far away from the body. The steady velocity potential is considered first. It is appropriate to decompose $\chi_{0}$ by

$$
\chi_{0}=-x+\chi
$$

where $\chi$ satisfies the Laplace equation in the fluid domain. At the free surface $\chi$ satisfies, to leading order in $U, \partial \chi / \partial z=0$. Furthermore, $\partial \chi / \partial n=n_{1}$ at $S_{B}$, where $\partial / \partial n$ denotes differentiation along the unit normal $n=\left(n_{1}, n_{2}, n_{3}\right)$ of the body surface, pointing out of the fluid, and $S_{B}$ denotes the mean position of the body. At the sea bottom $(z=-h)$ $\partial \chi / \partial z=0$, and in the far field $\left(\left(x^{2}+y^{2}\right)^{1 / 2} \rightarrow \infty\right), \nabla \chi \rightarrow 0$. The boundary value problem for $\chi$ is solved by applying a source distribution over $S_{B}$. The source Green function and the practical evaluation of it is described in the appendix.

\subsection{The linear potential}

Assuming time harmonic oscillations with frequency of encounter $\sigma, \Phi$ reads

$$
\Phi(\mathbf{x}, t)=\operatorname{Re}\left\{\phi(\mathbf{x}) e^{i \sigma t}\right\}=\operatorname{Re}\left\{\left[A \phi_{D}(\mathbf{x})+i \sigma \sum_{j=1}^{6} \xi_{j} \phi_{j}(\mathbf{x})\right] e^{i \sigma t}\right\}
$$

Here $A$ denotes the amplitude of the incoming waves, $\phi_{D}$ the diffraction potential, $\xi_{j}$ the amplitude of the body motion in the $j$ th mode (surge, sway, heave, roll, pitch and yaw, 
respectively), and $\phi_{j}$ the corresponding radiation potential for unit amplitude of motion. $\phi_{D}$ may properly be decomposed by

$$
\phi_{D}=\phi_{0}+\phi_{7}
$$

where

$$
\phi_{0}=\frac{i g}{\omega} \frac{\cosh K(z+h)}{\cosh K h} e^{-i K(x \cos \beta+y \sin \beta)}
$$

denotes the incoming wave potential and $\phi_{7}$ the scattering potential. $\omega$ denotes the orbital frequency of the incoming waves and $g$ the acceleration due to gravity. The wave angle $\beta$ is defined as the angle between the positive $x$-axis and the wave direction. The case $\beta=0$ corresponds to following waves, while $\beta=\pi$ corresponds to head waves. $K$ denotes the wave number of the incoming waves, and is related to the orbital frequency by the dispersion relation for gravity waves in water of finite depth, i.e.

$$
\omega^{2}=g K \tanh K h
$$

The relation between $\sigma, \omega, K$ and $U$ reads

$$
\sigma=\omega-U K \cos \beta
$$

The potentials $\phi_{j}, j=1, \ldots 7$, satisfy the Laplace equation, according to the previous assumptions. The free surface boundary condition reads

$$
-\nu \phi_{j}+2 i \tau \nabla_{1} \phi_{j} \cdot \nabla_{1} \chi_{\bullet}+i \tau \phi \nabla_{1}^{2} \chi_{\bullet}+\frac{\partial \phi_{j}}{\partial z}=0 \text { at } z=0, j=1, \ldots, 6, D
$$

where the subscript $D$ means the diffraction potential, $\nabla_{1}$ denotes the horizontal gradient, $\nu=\sigma^{2} / g$ and $\tau=U \sigma / g$. Far away from the body, where $\chi_{0}=-x,(8)$ reduces to

$$
-\nu \phi_{j}-2 i \tau \frac{\partial \phi_{j}}{\partial x}+\frac{\partial \phi_{j}}{\partial z}=0 \text { at } z=0, j=1, \ldots, 7
$$

The body boundary condition reads

$$
\frac{\partial \phi_{j}}{\partial n}=\left\{\begin{array}{cl}
n_{j}+\frac{U}{i v} m_{j}, & j=1, \ldots, 6 \\
-\frac{\partial \phi_{0}}{\partial n}, & j=7
\end{array} \quad \text { at } S_{B}\right.
$$

where $\left(n_{4}, n_{5}, n_{6}\right)=\mathbf{x} \times \mathbf{n},\left(m_{1}, m_{2}, m_{3}\right)=-\mathbf{n} \cdot \nabla\left(\nabla \chi_{s}\right)$ and $\left(m_{4}, m_{5}, m_{6}\right)=-\mathbf{n} \cdot \nabla\left(\mathbf{x} \times \nabla \chi_{s}\right)$. The boundary condition at the sea floor reads

$$
\frac{\partial \phi_{j}}{\partial z}=0 \text { at } z=-h, j=1, \ldots, 7
$$

The radiation condition in the far field, which may be obtained from the Green function discussed in $§ 3.1$, gives

$$
\phi_{j}=R^{-1 / 2} H_{j}(\theta) \frac{\cosh k_{1}(\theta)(z+h)}{\cosh k_{1}(\theta) h} e^{-i k_{1}(\theta) R\left(1+O\left(\tau^{2}\right)\right)}+\mathcal{O}\left(\frac{1}{R}\right) \quad \text { as } R \rightarrow \infty
$$

Here polar coordinates are introduced by $x=R \cos \theta, y=R \sin \theta$. The angle dependent wave number, $k_{1}(\theta)$, is given in $\S 3.1$ by eq. $(29)$. $H_{j}(\theta)$ denotes the amplitude distribution of $\phi_{j}$. 


\subsection{The second order potential $\psi^{(2)}$}

The second order potential $\psi^{(2)}$ (being Laplacian) enters in the equations for the mean second order fluid pressure, the mean drift force and mean drift moment only multiplied by $U$. To first order in $U$ it is then sufficient to consider the boundary value problem for $\psi^{(2)}$ at zero speed. Thus, the free surface boundary condition for $\psi^{(2)}$ satisfies

$$
\frac{\partial \psi^{(2)}}{\partial z}=-\frac{1}{g} \overline{\frac{\partial}{\partial t} \nabla \phi^{(1)} \cdot \nabla \phi^{(1)}}+\frac{1}{g^{2}} \frac{\overline{\partial \phi^{(1)}}}{\partial t} \frac{\partial^{3} \phi^{(1)}}{\partial z \partial t^{2}}+\frac{1}{g} \frac{\overline{\partial \phi^{(1)}} \frac{\partial^{2} \phi^{(1)}}{\partial t}}{\partial z^{2}}=-\frac{\sigma}{2 g} \operatorname{Im}\left(\phi \frac{\partial^{2} \phi^{*}}{\partial z^{2}}\right) \text { at } z=0
$$

where a bar denotes time average, a star complex conjungate, and we have applied $\phi^{(1)}=$ $\operatorname{Re}\left(\phi e^{i \sigma t}\right)$ for the first order potential (see eq. 3). Applying the results derived by Ogilvie ${ }^{10}$ for the complete second order potential, the body boundary condition reads

$$
\frac{\partial \psi^{(2)}}{\partial n}=V_{n}^{(2)} \quad \text { at } S_{B}
$$

where $V_{n}^{(2)}$

$$
V_{n}^{(2)}=-\overline{\mathbf{n} \cdot\left[\left(\xi^{(1)}+\alpha^{(1)} \times \mathbf{x}\right) \cdot \nabla\right] \nabla \phi^{(1)}}+\overline{\left(\alpha^{(1)} \times \mathbf{n}\right) \cdot\left[\frac{d}{d t}\left(\xi^{(1)}+\alpha^{(1)} \times \mathbf{x}\right)-\nabla \phi^{(1)}\right]}
$$

Here, $\xi^{(1)}=\operatorname{Re}\left\{\left(\xi_{1}, \xi_{2}, \xi_{3}\right) e^{i \sigma t}\right\}$ and $\alpha^{(1)}=\operatorname{Re}\left\{\left(\xi_{4}, \xi_{5}, \xi_{6}\right) e^{i \sigma t}\right\}$ denote respectively the first order translations and rotations of the body. Furthermore, $\partial \psi^{(2)} / \partial z=0$ at $z=-h$, and $\nabla \psi^{(2)} \rightarrow 0$ for $\left(x^{2}+y^{2}\right)^{1 / 2} \rightarrow \infty$.

\section{Solution of the time harmonic problems}

\subsection{The wave green function}

The radiation and diffraction problems will be solved by applying Green's second identity to the entire fluid domain. As Green function we apply a pulsating source translating with small forward speed satisfying (9), (11) and the appropriate radiation condition. This function reads

$$
G(\mathbf{x}, \xi)=\frac{1}{r_{1}}+\frac{1}{r_{2}}+\Psi(\mathbf{x}, \xi)
$$

where

$$
r_{1}=\sqrt{(x-\xi)^{2}+(y-\eta)^{2}+(z-\zeta)^{2}}
$$

and

$$
r_{2}=\sqrt{(x-\xi)^{2}+(y-\eta)^{2}+(z+\zeta+2 h)^{2}}
$$

The wave part of the source potential, obtained by use of Fourier transform, reads

$$
\begin{array}{r}
\Psi(\mathbf{x}, \boldsymbol{\xi})=\frac{1}{2 \pi} \int_{0}^{2 \pi} \int_{0}^{\infty} B(k, \alpha) \frac{2 e^{-k h}}{\cosh k h} \cosh k(\zeta+h) \cosh k(z+h) \\
\times e^{i k((x-\xi) \cos \alpha+(y-\eta) \sin \alpha)} d k d \alpha
\end{array}
$$


where

$$
B(k, \alpha)=\frac{\nu+k(1-2 \tau \cos \alpha)}{k(\tanh k h+2 \tau \cos \alpha)-\nu}
$$

The path of integration, which is determined by use of Rayleigh viscosity, is above the pole at $k=\kappa_{1}$. This also determines the radiation condition in the far field. The pole $\kappa_{1}$ is determined by

$$
\kappa_{1}\left(\tanh \kappa_{1} h+2 \tau \cos \alpha\right)-\nu=0
$$

For small values of $\tau$ we may expand $B$ in a power series by

$$
B(k, \alpha)=B^{0}(k, \alpha)+\tau B^{1}(k, \alpha)+O\left(\tau^{2}\right)
$$

We find that $B^{0}$ and $B^{1}$ are given by

$$
\begin{gathered}
B^{0}(k, \alpha)=\frac{\nu+k}{k \tanh k h-\nu} \\
B^{1}(k, \alpha)=2 i^{2} k \cos \alpha\left(\frac{1}{k \tanh k h-\nu}+\frac{(\nu+k)}{(k \tanh k h-\nu)^{2}}\right)=2 i^{2} k \cos \alpha \frac{\partial B^{0}}{\partial \nu}
\end{gathered}
$$

Inserting (24) into (19) it is easily seen that $G$, following the idea of Huismans and Hermans ${ }^{11}$ in the infinite depth case, may be expanded in an asymptotic series provided that $\tau<<1$, i.e.

$$
G=G^{0}+\tau G^{1}+O\left(\tau^{2}\right)
$$

where $G^{0}$ denotes the Green function for $\tau=0$ (but keeping $\nu$ ) and $G^{1}$ is given by

$$
G^{1}=2 i \frac{\partial^{2} G^{0}}{\partial \nu \partial x}
$$

The far-field behaviour of $G$, which will be used in the computations of the forces, is obtained by applying contour integration and the method of stationary phase, giving

$$
G(R, \theta, z ; \xi, \eta, \zeta)=R^{-1 / 2} \tilde{h}(\xi, \theta) \frac{\cosh k_{1}(\theta)(z+h)}{\cosh k_{1}(\theta) h} e^{i k_{1}(\theta) R \cos \left(\alpha_{0}-\theta\right)}
$$

where the stationary phase angle $\alpha_{0}(\theta)$ is given implicitly by

$$
\sin \left(\alpha_{0}-\theta\right)=\frac{2 \tau}{C_{g}\left(k_{1} h\right)} \sin \theta, \cos \left(\alpha_{0}-\theta\right)<0
$$

and $k_{1}(\theta)$ by

$$
k_{1}\left(\tanh k_{1} h+2 \tau \cos \alpha_{0}\right)-\nu=0
$$

$C_{g}\left(k_{1} h\right)$ reads

$$
C_{g}\left(k_{1} h\right)=\tanh k_{1} h+\frac{k_{1} h}{\cosh ^{2} k_{1} h} \equiv \frac{d \omega_{1} / d k_{1}}{\frac{1}{2} g / \omega_{1}}
$$

where $\omega_{1}$ denotes the orbital frequency of the wave with wave number $k_{1}$, and is given by $\omega_{1}^{2}=g k_{1} \tanh k_{1} h$. Thus, $C_{g}\left(k_{1} h\right)$ denotes the ratio between the group velocity of a wave with frequency $\omega_{1}$ at moderate and infinite water depth, respectively. 
For small value of $\tau$ we may obtain $\alpha_{0}(\theta)$ and $k_{1}(\theta)$ explicitly by

$$
\begin{gathered}
\alpha_{0}=\pi+\theta-2 \tau^{h} \sin \theta \\
k_{1}(\theta)=k_{0}\left(1+2 \tau^{h} \cos \theta\right)
\end{gathered}
$$

where

$$
\tau^{h}=\frac{\tau}{\tanh K h+\frac{K h}{\cosh ^{2} K h}}=\frac{\tau}{C_{g}(K h)}
$$

and $k_{0}$ is determined by

$$
k_{0} \tanh k_{0} h=\nu=\frac{\sigma^{2}}{g}
$$

The amplitude $\tilde{h}(\xi, \theta)$ of $(27)$ reads

$$
\tilde{h}(\xi, \theta)=\sqrt{\frac{2 \pi}{k_{0}}} \frac{k_{1}\left(\tanh k_{1} h+1\right)}{C_{g}\left(k_{1} h\right)}\left(e^{k_{1} \zeta}+e^{-k_{1}(\zeta+2 h)}\right) e^{-i k_{1}\left(\xi \cos \alpha_{0}+\eta \sin \alpha_{0}\right)-i \pi / 4}+\mathcal{O}\left(\tau^{2}\right)
$$

In the limit $h \rightarrow \infty$ the expressions for $\alpha_{0}, k_{1}$ and $\bar{h}$ become identical to the results derived by Nossen et al. ${ }^{5}$ assuming deep water. As eqs. (31) - (35) show, $C_{g}(K h)$ and $\tanh K h$ are measures of the effect of $h$ on the diffracted and radiated waves. Figure 1 illustrates how the wave characteristics at moderate depth deviates from the characteristics at deep water. This deviation is less than $5 \%$ when $K h>2$, and at most $15 \%$ when $1<K h<2$. The current effect on the wave characteristics is significantly enhanced when $K h<1$, however.

\subsection{Integral equations}

The boundary value problems are then solved by applying Green's theorem to $G$, defined by (16), and $\phi_{D}$ in the diffraction problem, and $G$ and $\phi_{j}$ in the radiation problem. The procedure follows the same steps as for $h=\infty$ (Nossen et al. ${ }^{5}$ eqs. 31-36). The only difference from the infinite depth case is that $G, \phi_{D}$ and $\phi_{j}$ now obey the rigid wall condition at $z=-h$, instead of being vanishing functions as $z \rightarrow-\infty$. Assuming that the body is wall-sided at the water line, and omitting terms of smaller order than $\tau$, we obtain for the diffraction problem

$$
\iint_{S_{B}} \phi_{D} \frac{\partial G}{\partial n} d S-2 i \tau \iint_{S_{F}} \phi_{D}\left(\nabla_{1} G \cdot \nabla_{1} \chi+\frac{1}{2} G \nabla_{1}^{2} \chi\right) d S-4 \pi \phi_{0}=\left\{\begin{array}{l}
-4 \pi \phi_{D}(\mathbf{x}) \\
-2 \pi \phi_{D}(\mathbf{x})
\end{array}\right.
$$

where in the first case $\mathbf{x}$ is in the fluid domain and in the second case $\mathbf{x}$ is on $S_{B} . S_{F}$ denotes the free surface. Similarly, we obtain for the radiation problem

$$
\iint_{S_{B}}\left(\phi_{j} \frac{\partial G}{\partial n}-G\left(n_{j}+\frac{U}{i \sigma} m_{j}\right)\right) d S-2 i \tau \iint_{S_{F}} \phi_{j}\left(\nabla_{1} G \cdot \nabla_{1} \chi+\frac{1}{2} G \nabla_{1}^{2} \chi\right) d S=\left\{\begin{array}{l}
-4 \pi \phi_{j}(\mathbf{x}) \\
-2 \pi \phi_{j}(\mathbf{x})
\end{array}\right.
$$

for $j=1, \ldots, 6$. 
For $\tau<<1$ we may for bounded values of the geometrical coordinate introduce the asymptotic expansion (25) for $G$ and

$$
\phi=\phi^{0}+\tau \phi^{1}
$$

for $\phi$. Substituting (25) and (38) into (36) - (37) we arrive at integral equations where the potentials $\phi^{0}$ and $\phi^{1}$ appear as unkowns on the wetted body surface only, see Nossen et al. ${ }^{5}$. The integral equations are solved by a conventional panel method where the body is discretized by quadrilateral panels. The integrals over the free surface appear only as quadratur. The singular parts of the Green function are integrated analytically over each panel. We find that the singularities in the wave part of the finite depth Green function with forward speed are the same as the singularities when the water depth is infinite. It is essential to return to the unperturbed equations (36) and (37) when we evaluate the far field behaviour of the potentials.

\section{The linear forces}

\subsection{The added mass and damping}

The pressure force and moment in the radiation problem is then obtained by applying the Bernoulli equation, giving

$$
F_{j}=\operatorname{Re}\left(-i \sigma e^{i \sigma t} \sum_{k=1}^{6} \xi_{k} f_{j k}\right), \quad j=1, \ldots, 6
$$

where the complex force coefficients read

$$
f_{j k} \equiv i \sigma a_{j k}+b_{j k}=\rho \iint_{S_{B}}\left(i \sigma \phi_{k}+U \nabla \chi_{\bullet} \cdot \nabla \phi_{k}\right) n_{j} d S
$$

Here $a_{j k}$ denote the added mass coefficients and $b_{j k}$ the damping coefficients. Timman and Newman ${ }^{12}$ showed, neglecting the $\chi$-field, that $f_{j k}$ satisfy the following symmetry

$$
f_{j k}(U)=f_{k j}(-U)
$$

Wu and Eatock-Taylor ${ }^{13}$ and Nossen et al. ${ }^{5}$ show that (41) is valid also for bodies of arbitrary shape when the $\chi$-field is not small. The restrictions in their proofs are that $U$ is small, the body is wall sided at the water line and the water depth is infinite.

It is easy to generalize the proof by Nossen et al. ${ }^{5}$, eqs. 50-53, and show that (41) is valid also when the water depth is arbitrary. One result of $(41)$ is that the diagonal terms $f_{k k}$ do not depend on the forward speed when this is small. Furthermore, (41) provides a check on the numerical code. As examples we show in figures $2 \mathrm{a}-\mathrm{c}$ the added mass coefficients $a_{13}$ and $a_{31}$ for a half immersed sphere of radius $a$ at Froude number $F r=U / \sqrt{g a}= \pm 0.04$, and three different water depths. $F r= \pm 0.04$ corresponds to $U= \pm 0.4 \mathrm{~ms}^{-1}$ when the radius is $a=10 \mathrm{~m}$. For zero forward speed we have that $a_{13}=a_{31}=0$ due to symmetry. These added mass coefficients are thus proportional to the forward speed when this is 
nonzero. The numerical results for $a_{13}(U)$ and $a_{31}(-U)$ illustrates the validity of $(41)$ and the accuracy of the numerical panel method. We observe that $a_{13}$ and $a_{31}$ are increased considerably when the water depth is reduced from $h / a=2$ to $h / a=1.2$. A small relative difference between $a_{13}$ and $a_{31}$ is revealed in figure 2c. This relative difference is expected to vanish by increasing the number of panels sufficiently, and is discussed further in $\S 6$.

\subsection{The exciting forces and moments}

The exciting forces and moments, obtained by pressure integration at the body, read

$$
F_{j}=-\rho \iint_{S_{B}}\left(\frac{\partial \Phi}{\partial t}+U \nabla \chi_{\bullet} \cdot \nabla \Phi\right) n_{j} d S \equiv \Re\left(A e^{i \sigma t} X_{j}\right), \quad j=1, \ldots, 6
$$

Here, $\Phi$ is given by (3) with $\xi_{j}=0, j=1, \ldots, 6$. The exciting forces and moments may as an alternative to direct pressure integration be obtained by generalized Haskind relations. With this method $X_{j}$ is found without solving the diffraction problem. Furthermore, obtaining the exciting forces and moments by two different methods provides an additional check of the analysis and the numerical code. The Haskind relations are derived by first introducing the reversed-flow radiation potentials $\psi_{j}, j=1, \ldots, 6$, satisfying the boundary conditions (8) and (10) with $U$ replaced by $-U$, but keeping the frequency of encounter. Nossen at al. ${ }^{5}$, eq. 62 , show for $h=\infty$ that $X_{j}$ may be expressed by

$$
X_{j}=i \sigma \rho \iint_{S_{\infty}}\left(\phi_{0} \frac{\partial \psi_{j}}{\partial n}-\psi_{j} \frac{\partial \phi_{0}}{\partial n}\right) d S+2 \sigma \tau \rho \int_{C_{\infty}} \phi_{0} \psi_{j} d y
$$

Here $S_{\infty}$ denotes a vertical circular cylinder enclosing the fluid at infinity and $C_{\infty}$ the water line of $S_{\infty}$. It may be shown that (43) is valid also when $h<\infty$. (43) may be further developed by introducing (5) for $\phi_{0}$ and the far field form of $\psi_{j}$, which may be obtained from (12). By carrying out the integration and neglecting terms being $O\left(\tau^{2}\right)$ we obtain

$$
X_{j}=\frac{\rho g \sigma}{\omega K} \sqrt{2 \pi k_{0}} C_{g}(K h) H_{j}^{-}\left(\beta+\pi+2 \tau^{h} \sin \beta\right) e^{i \pi / 4}
$$

Here, $H_{j}^{-}$denotes the amplitude distribution of $\psi_{j}$, obtained form $H_{j}$ by replacing $\tau$ by $-\tau$ (but keeping $\nu$ ). $H_{j}$ is found by introducing (12) in (37), which gives

$$
\begin{aligned}
H_{j}(\theta)= & -\frac{1}{4 \pi} \iint_{S_{B}}\left(\phi_{j} \frac{\partial \tilde{h}}{\partial n}-\left(\tilde{h}-\frac{\tau}{i \nu} \nabla \chi_{s} \cdot \nabla \tilde{h}\right) n_{j}\right) d S \\
& +\frac{i \tau}{2 \pi} \iint_{S_{F}} \phi_{j}\left(\nabla_{1} \tilde{h} \cdot \nabla_{1} \chi+\frac{1}{2} \tilde{h} \nabla_{1}^{2} \chi\right) d S
\end{aligned}
$$

where $\tilde{h}(\xi, \theta)$ is given by (35).

We then examine the exciting forces on the half-immersed sphere. In the first example we assume that the waves are propagating orthogonal to the forward speed direction, i.e. $\beta=\pi / 2$. Due to the symmetry in this example, both the sway force, $X_{2}$, and the heave force, $X_{3}$, are unaffected by a small forward speed, and are thus given by the values at 
zero speed. The surge exciting force, $X_{1}$, which vanishes for $U=0$, is proportional to the forward speed, however. $X_{1}$ is shown in figure 3 for $\operatorname{Fr}=0.04$ at $h / a=1.2$ and $h=\infty$ by both the generalized Haskind relations and by pressure integration. The agreement between the two methods is excellent. The figure illustrates furthermore that $X_{1}$ is smaller for $h / a=1.2$ than for $h=\infty$. Results for $h / a=2$ (not shown) are very close to those for $h=\infty$.

Next we consider incoming head waves, i.e. $\beta=\pi$. Results for $X_{1}$ and $X_{3}$ are shown in figures $4 \mathrm{a}-\mathrm{b}$ for $\mathrm{Fr}= \pm 0.04$ at $h / a=1.2$ and $h=\infty$. We observe that $X_{1}$ and $X_{3}$ are slightly increased due to a reduced water depth. Results for $h / a=2$ (not shown) fall in between the curves for $h / a=1.2$ and $h=\infty$. In figures 5a-b are displayed the change in $X_{1}$ and $X_{3}$ with respect to the forward speed, as function of the water depth and wave number. Both the generalized Haskind relations and pressure integration are applied, with an excellent agreement in the numerical results. We observe that the values of $\partial X_{3} / \partial U$ are considerably enhanced for a decreasing water depth when the wave length is moderate and long $(K h<1)$. The opposite is true for $\partial X_{1} / \partial U$.

\section{The mean drift force and moment}

\subsection{The mean horizontal drift force}

The mean horizontal drift force, F, may be obtained by evaluating the linear momentum flux at a control surface some distance away from the body. As control surface the vertical circular cylinder at infinity, $S_{\infty}$, is applied. Thereby the horizontal drift force may be expressed by the far field amplitudes of the radiated and diffracted waves. By applying the Bernoulli equation and conservation of mass, Grue and Palm ${ }^{14}$ show that $\mathbf{F}$ reads

$$
\begin{aligned}
\mathbf{F}=\frac{\rho}{2 g} \int_{C_{\infty}}\left(\overline{\left(\frac{\partial \Phi}{\partial t}\right)^{2}}-U^{2} \overline{\left(\frac{\partial \Phi}{\partial x}\right)^{2}}\right) \mathbf{n} d s-\rho \iint_{S_{\infty}}\left(\frac{1}{2} \overline{(\nabla \Phi)^{2}} \mathbf{n}-\overline{\nabla \Phi \frac{\partial \Phi}{\partial n}}\right) d S \\
+\rho U \mathbf{j}\left(\int_{C_{\infty}} \overline{\zeta^{(1)} \frac{\partial \Phi}{\partial s}} d s+\iint_{S_{\infty}} \frac{\partial \psi^{(2)}}{\partial s} d s d z\right)
\end{aligned}
$$

which is valid for arbitrary values of $h$ and $U$. Here $s$ denotes the arclength for a constant level circel and $\zeta^{(1)}$ the first order free surface elevation. Assuming that there is no circulation in the fluid, the contribution from $\psi^{(2)}$ disappears. Furthermore, using the far field properties of the potentials it may be shown that $\int_{C_{\infty}} \overline{\zeta^{(1)} \partial \Phi / \partial s} d s$ is $O\left(\tau^{2}\right)$. Thus, omitting terms being $O\left(\tau^{2}\right)$, we obtain

$$
\mathbf{F}=\frac{\rho}{2 g} \int_{0}^{2 \pi} \overline{\left(\frac{\partial \Phi}{\partial t}\right)_{z=0}^{2}} R d \theta-\rho \int_{0}^{2 \pi} \int_{-h}^{0}\left(\frac{1}{2} \overline{(\nabla \Phi)^{2}} \mathbf{n}-\overline{\nabla \Phi \frac{\partial \Phi}{\partial n}}\right) R d z d \theta
$$

where $\theta$ denotes the polar angle. Introducing the far field form of the velocity potential and carrying out the integrals we obtain for $F=\left(F_{x}, F_{y}\right)$ after some algebra

$$
\frac{F_{x}}{\rho g A^{2}}=-\frac{g k_{0}}{4 \omega^{2}}\left\{\int_{0}^{2 \pi}\left(C_{g}\left(k_{1} h\right) \cos \theta+2 \tau \sin ^{2} \theta\right)|H(\theta)|^{2} d \theta+2 C_{g}(K h) \cos \beta R e[S]\right\}
$$




$$
\frac{F_{y}}{\rho g A^{2}}=-\frac{g k_{0}}{4 \omega^{2}}\left\{\int_{0}^{2 \pi}\left(C_{g}\left(k_{1} h\right) \sin \theta-2 \tau \sin \theta \cos \theta\right)|H(\theta)|^{2} d \theta+2 C_{g}(K h) \sin \beta R e[S]\right\}
$$

where

$$
\begin{gathered}
S=\sqrt{\frac{2 \pi}{k_{0}}} e^{i \pi / 4} H^{*}\left(\beta+2 \tau^{h} \sin \beta\right) \\
\frac{i g}{\omega} H(\theta)=H_{7}(\theta)+i \sigma \sum_{j=1}^{6} \frac{\xi_{j}}{A} H_{j}(\theta)
\end{gathered}
$$

(A star means complex conjungate.) $H_{j}(\theta)$ is given by (45), and $H_{7}(\theta)$ by

$$
H_{7}(\theta)=-\frac{1}{4 \pi}\left\{\iint_{S_{B}} \phi_{D} \frac{\partial h}{\partial n} d S-2 i \tau \iint_{S_{F}} \phi_{D}\left(\nabla_{1} h \cdot \nabla_{1} \chi+\frac{1}{2} h \nabla_{1}^{2} \chi\right) d S\right\}
$$

\subsection{The mean vertical drift moment}

The mean vertical drift moment, $M_{z}$, may be obtained by evaluating the angular momentum flux in the far field. By using that the contribution from the pressure field vanishes, $M_{z}$ reads

$$
\begin{aligned}
& M_{z}=-\rho \iint_{S_{\infty}} \overline{\frac{\partial \Phi}{\partial \theta} \frac{\partial \Phi}{\partial R}} d S-\rho U \int_{C_{\infty}} \overline{\left(y \frac{\partial \Phi}{\partial R}-\frac{x}{R} \frac{\partial \Phi}{\partial \theta}\right) \zeta^{(1)}} d s \\
& -\rho U \iint_{S_{\infty}}\left(y \frac{\partial \psi^{(2)}}{\partial R}-\frac{x}{R} \frac{\partial \psi^{(2)}}{\partial \theta}\right) d S+\rho U^{2} \int_{C_{\infty}} y n_{1} \overline{\zeta^{(2)}} d s
\end{aligned}
$$

where $\zeta^{(2)}$ denotes the second order free surface elevation. (53) is valid for arbitrary values of $U$ and $h$. The last term will, however, be disregarded here being $O\left(\tau^{2}\right)$.

We observe that the two first terms of (53) are entirely determined by the first order potential. Introducing the far field expression for $\Phi$ and carrying out the integrals, we obtain for these two terms after some algebra

$$
\begin{aligned}
& \frac{M_{z}^{(1)}}{\rho g A^{2}}=-\frac{g}{4 \omega^{2}} \int_{0}^{2 \pi}\left(C_{g}\left(k_{1} h\right)-2 \tau \cos \theta\right) \operatorname{Im}\left[H \frac{\partial H^{*}}{\partial \theta}\right] d \theta \\
- & \frac{g}{2 \omega^{2}}\left\{\left(1-\frac{K}{C_{g}(K h)} \frac{d C_{g}(K h)}{d K}\right) \tau \sin \beta \operatorname{Im}[S]+\left(C_{g}(K h)-2 \tau \cos \beta\right) \operatorname{Im}\left[S^{\prime}\right]\right\}
\end{aligned}
$$

where $S$ is given by (50) and

$$
S^{\prime}=\sqrt{\frac{2 \pi}{k_{0}}} e^{i \pi / 4}\left[H^{\prime}\left(\beta+2 \tau^{h} \sin \beta\right)\right]^{*}
$$

Here a prime denotes differentiation with respect to the argument.

The third term of (53) may be recast in a form where $\psi^{(2)}$ is expressed by the first order fluid motions and body responses. Using the procedure of Grue and Palm ${ }^{14}$ for $h=\infty$, we can show for $h<\infty$ that

$$
M_{z}^{(2)}=-\rho U \iint_{S_{\infty}}\left(y \frac{\partial \psi^{(2)}}{\partial R}-\frac{x}{R} \frac{\partial \psi^{(2)}}{\partial \theta}\right) d S=-\rho U \iint_{S_{P}+S_{B}} \Psi_{s} \frac{\partial \psi^{(2)}}{\partial n} d S
$$


Here $\Psi_{\text {, }}$ is a Laplacian velocity potential satisfying $\partial \Psi_{\mathrm{s}} / \partial z=0$ for $z=0, z=-h$, $\partial \Psi_{s} / \partial n=0$ on $S_{B}$ and $\Psi_{s}=-y$ for $\left(x^{2}+y^{2}\right)^{1 / 2} \rightarrow \infty$. We note that $\Psi_{s}$ is the steady potential if the body is moving along the positive $y$-axis (corresponding to $\chi_{\text {, when }}$ whe body is moving along the positive $x$-axis). Using (13) and (14) for $\partial \psi^{(2)} / \partial n$ at $S_{B}$ and $S_{F}$, we obtain

$$
\frac{M_{z}^{(2)}}{\rho g A^{2}}=\frac{\tau}{2 g A^{2}} \iint_{S_{F}} \Psi, I m\left(\phi \frac{\partial^{2} \phi^{*}}{\partial z^{2}}\right) d S-\frac{\tau}{\sigma A^{2}} \iint_{S_{B}} \Psi_{s} V_{n}^{(2)} d S
$$

where $V_{n}^{(2)}$ is given by (15). The total moment with small forward speed, given by $M_{z}=$ $M_{z}^{(1)}+M_{z}^{(2)}$, is by (54) - (57) entirely determined by products between first order quantities.

A complete discussion of the $\psi^{(2)}$-field for infinite water depth is provided by Grue and Palm ${ }^{14}$. The solution of $\psi^{(2)}$ for finite water depth is easily obtained by using Green's theorem to $\psi^{(2)}$ and the Green function given by (64) in the appendix.

\subsection{Numerical results for $F_{x}$}

Numerical results for $F_{x}, F_{y}$ and $M_{z}$ for floating bodies in oblique waves at infinite water depth have been presented earlier, see e.g. Grue ${ }^{6}$, Grue and Palm ${ }^{1516}$. Our main interest here is the effect of the restricted water depth on the drift forces and the wave drift damping. At zero speed

$$
\frac{g K}{\omega^{2}} C_{g}(K h)=1+\frac{2 K h}{\sinh K h}
$$

appears as prefactor in (48), (49) and (54). This function has a value between 1.5 and 2 for $K h<1$, but becomes rapidly close to unity when $K h>1$. The forward speed part of (48), (49) and (54) has the prefactor

$$
\frac{\tau g k_{0}}{\omega^{2}} \simeq \frac{\tau}{\tanh K h}
$$

An amplification factor of 2 then occurs for $K h=0.5$, for example. The mean horizontal drift force and vertical moment are also dependent on $H(\theta)$ being a function of $h / T$ and $K h$, which is not known explicitly. The numerical results indicate, however, that the prefactors (58) - (59) give the variation in $F$ and $M_{z}$ with respect to $h$, roughly speaking.

We shall confine ourselves to study numerical results for $F_{x}$ in head seas only. Results for $F_{x}, F_{y}$ and $M_{z}$ in oblique waves will be presented elsewhere. Three different bodies will be considered; a half-immersed sphere, a vertical truncated cylinder and a ship. For small forward speed we may write $F_{x}$ in the form

$$
F_{x}=F_{x 0}+\operatorname{Fr} F_{x 1}
$$

where $F_{x 0}$ denotes the drift force at zero speed and $F r F_{x 1}$ gives the change in $F_{x}$ due to the forward speed. The factor $F_{x 1}$ denotes the wave drift damping coefficient. Let us then consider the mean drift force on the half-immersed sphere, which for the moment is assumed to be restrained from responding to the waves. In figures $6 a-b$ results for $F_{x}$ are shown for incoming head waves and $F r=U / \sqrt{g a}= \pm 0.04$ at three different water depths. 
Figure $6 a$ shows that we obtain practically speaking the same drift force for $h / a=2$ as for $h=\infty$. $F_{x}$ is, however, increased by about $25 \%$ when the water depth is reduced to $h / a=1.2$. In figure $6 \mathrm{c}$ is displayed the wave drift damping coefficient for the sphere, which is obtained from the results in figures $6 \mathrm{a}-\mathrm{b}$. We observe that $F_{x 1}$ is slightly larger for $h / a=1.2$, and slightly smaller for $h / a=2$, than for $h=\infty$.

The next geometry is a vertical truncated cylinder with radius and draught $a$, which also is assumed to be restrained. Results for $F_{x 0}$ and $F_{x 1}$ are shown in figures $7 \mathrm{a}-\mathrm{b}$ for incoming head waves when the water depth is either $h / a=1$ (the cylinder is spanning the entire fluid layer) or $h=\infty$. A larger effect of a finite $h$ occurs in this example than in the previous. This is partly due to that the geometries are different, but also that an appreciable value of the force occurs at smaller $K h$ for the cylinder (when $h / a=1$ ), where the prefactors (58) - (59) are larger, than for the sphere $(h / a=1.2)$.

In the next examples we consider the mean drift force and the wave drift damping for a ship with length $L=230 \mathrm{~m}$, beam $B=41 \mathrm{~m}$ and draught $T=15 \mathrm{~m}$. The ship can advance with a small speed while responding freely to incoming head waves. A discretization of the ship is shown in Grue ${ }^{6}$. Results for $F_{x 0}$ and $F_{x 1}$ are shown in figures 8a-b for water depth $h=60 \mathrm{~m}$ and in figures $9 \mathrm{a}-\mathrm{b}$ for $h=30 \mathrm{~m}$. The corresponding results for $h=\infty$ are indicated for comparison. We observe that both the zero speed force and the wave drift damping coefficient differs only slightly from the corresponding values for deep water and that $F_{x 0}$ is the same for $h=60 m$ as for $h=\infty$. The results for $h=60 m$ are not surprising. We remark that appreciable values of $F_{x 0}$ and $F_{x 1}$ occur for $K L>6$, which means that $K h>1.6$. According (58) - (59) a minor effect of the water depth is then expected. For $h=30 m$ and $K L>6$ we have that $K h>0.8$. We would then expect an enhancement in the force compared to $h=\infty$. The numerical results show that this is not true, however.

The water depth is then reduced to $h=18 \mathrm{~m}$. This means a ratio between the water depth and draught of the ship being $h / T=1.2$, which is a very shallow condition. This condition corresponds to one of the examples in Pinkster and Huismans ${ }^{9}$, fig. 14., who consider the mean drift force at zero forward speed for a ship being slightly different from ours. Their study, being both theoretical and experimental, is performed with wave amplitudes up to $13 \%$ of the water depth in the experiments. Our results for $F_{x 0}$ at $h / T=1.2$, shown in figure 10a, agree both qualitatively and quantitatively with their results. We note that there is a pronounced peak in $F_{x 0}$ for $K L \sim 6-7$, which is not observed for larger water depth. Next we show in figure $10 \mathrm{~b}$ the wave drift damping coefficient, $F_{x 1}$, for $h / T=1.2$. Most notably is the peak at $K L \sim 6$, with a value being about three times larger than for $h=30 m(h / T=2)$. This means that a considerable enhancement of the wave drift damping occurs when the wave length is comparable to the ship length and the depth to draught ratio is slightly larger than unity. For $K L>9$, however, the wave drift damping is the same as in deeper water, practically speaking.

\section{Convergence}

The mid-point rule, used in large parts of the numerical integration, introduce global errors in the potentials and the forces being proportional to $1 / N_{B}$ and $1 / N_{F}$, where $N_{B}$ and $N_{F}$ 
denote the number of panels on $S_{B}$ and $S_{F}$, respectively. Thus, it is expected that the numerical errors are reduced when $1 / N_{B}$ and $1 / N_{F}$ both go to zero. First we consider the numerical errors in the added mass coefficients, $a_{13}$ and $a_{31}$, for the half-immersed sphere at $F r=0.04$ and $h / a=1.2$, which were shown in figure 2c. Here we consider $a_{13}$ and $a_{31}$ as function of $1 / N_{B}$ (and $1 / N_{F}$ ) for fixed value of the frequency, with results shown in figure 11. The exact values of $a_{13}$ and $a_{31}$ satisfy the Timman-Newman relations (41). The numerical results, on the the other hand, exhibit a discrepancy between $a_{13}$ and $a_{31}$ which does not decay until $1 / N_{B}$ becomes smaller than 0.0025 . Then the results indicate convergence. The smallest relative discrepancy between $a_{13}$ and $a_{31}$ in the figure is $4 \%$. This number indicates how large the relative numerical error is.

Next we consider the numerical error in the mean drift force for the half-immersed sphere. The examples displayed in figures 12a-b indicate that the relative numerical error is proportional to $1 / N_{B}$ (and $1 / N_{F}$ ) and is less than about 5 per mille for the finest discretization. We observe that the relative error is the same for a small forward speed as for $U=0$. Convergence of the wave drift damping coefficient, $F_{x 1}$, is shown in figure 12c. The curve indicates that the wave drift damping is predicted with the same accuracy as the drift force at zero speed.

\section{Balance of energy}

Balance of energy relates the mean work due to the pressure force on the body to the mean energy flux at a surrounding control surface, assuming that there is no dissipation in the fluid. This provides a check on the whole model and the numerical method. In the cases when the body is restrained or freely floating in the waves, no work is performed by the pressure force. This means that the mean energy flux at the control surface is zero. Let us consider the mean energy flux at $S_{\infty}$ which is given by

$$
W=\overline{\iint_{S_{\infty}}\left(p+\frac{1}{2} \rho|\mathbf{v}|^{2}+\rho g z\right) \mathbf{v} \cdot \mathbf{n} d S}
$$

Applying conservation of mass and the Bernoulli equation for the pressure we have

$$
W=-\rho \iint_{S_{\infty}} \frac{\overline{\partial \Phi} \frac{\partial \Phi}{\partial t}}{\partial n} d S+\rho U \int_{C_{\infty}} \overline{\frac{\partial \Phi}{\partial t} \zeta^{(1)}} n_{1} d l
$$

We note that $W$ is entirely determined by products between first order quantities. Inserting for the velocity potential we obtain for $W$, omitting terms being $O\left(\tau^{2}\right)$

$$
\frac{W}{2 E c_{g}}=\frac{\sigma}{2 \omega C_{g}(K h)} \int_{0}^{2 \pi}\left(C_{g}\left(k_{1} h\right)-2 \tau \cos \theta\right)|H(\theta)|^{2} d \theta+\frac{\sigma k_{0}}{\omega K} \cos \beta R e(S)
$$

where $H(\theta)$ is given by (50) and $S$ by (51). Furthermore, $E \equiv \frac{1}{2} \rho g A^{2}$ and $c_{g}$ denote the mean energy density and the group velocity of the incoming waves, respectively. As example we consider in figure 13 the energy flux at $S_{\infty}$ due to the restrained sphere for values of the Froude number between -0.08 and 0.08 . The normalized energy flux is 
obviously a nonlinear function of the Froude number, having a minimum of $-1.4 \times 10^{-4}$ at $F r=0$, and a maximum of about 0.065 at $F r=0.08$. The significant values of $W$ for nonzero Froude number are partly due to numerical errors and partly because the theory is consistent only to leading order in the Froude number. By assuming that the part of the energy flux, which is not appropriately accounted for by the theory, may be represented by $\sum_{n=2}^{5} \alpha_{n} F r^{n}$, we may apply the method of least squares to find $\alpha_{n}$. We obtain that $\alpha_{2}=4.1, \alpha_{3}=24.5, \alpha_{4}=235$ and $\alpha_{5}=3943$. By subtraction

$$
\frac{W^{\prime}}{2 E c_{g}}=\frac{W}{2 E c_{g}}-\sum_{n=2}^{5} \alpha_{n} F r^{n}
$$

The values of $W^{\prime}$, also shown in figure 13, are found to be of the same order of magnitude as the zero speed value of $W$. This demonstrates that the numerical errors in the model for small forward speed are of the same order of magnitude as for $U=0$.

\section{Conclusion}

We have studied interaction between waves and floating bodies with small speed, with special attention to the effect of a finite water depth. Two new parameters then enters into the problem, viz. the ratio between the water depth and the draught of the body, $h / T$, and the wave number nondimensionalized by the water depth, $K h$.

The ratio between the group velocity at moderate and infinite water depth appears in the formulas for the scattered waves by the body and in the far field formulas for the forces. This ratio, together with the value of $\tanh K h$, indicates how much a limited value of the water depth changes the forces from the corresponding results in deep water. We find that significant changes occurs when $K h<1$ and $h / T$ is close to unity.

Of particular interest is the prediction of the wave drift damping which is obtained from the mean horizontal drift force being a function of the forward speed. Numerical results are presented for a half-immersed sphere, a vertical truncated cylinder and a ship. The wave drift damping for the ship may be three times larger than the corresponding result in deep water when the wave length is comparable to the ship length and the ratio between the water depth and the ship draught is slightly larger than unity. The wave drift damping for the same ship is, however, very close to the results in deep water when $h / T>2$.

The problem is solved consistently to leading order in the wave amplitude and the forward speed by means of integral equations. The mean second order horizontal force and vertical moment are given consistently to second order in the wave amplitude. The velocity potential appears as unknown only on the wetted body surface. A discretization of the free surface is needed for quadratur, however. A panel method which is applicable to threedimensional bodies of arbitrary shape is exploited as numerical tool. The computational burden is comparable to the zero speed problem.

Integral checks like the generalized Timman-Newman relations, the generalized Haskind relation and conservation of energy demonstrates that the forces are predicted with the same accuracy to leading order in the forward speed as for zero speed. Convergence of the 
method with respect to the discretization of the wetted body surface and the free surface is discussed.

This research was supported by the Joint Industry Project "Wave drift damping". The WAMIT radiation/diffraction program has been provided by Det Norske Veritas Sesam Systems.

\section{A Appendix. The Green function for the steady problem}

For convenience we apply hereafter geometrical coordinates non-dimensionalized by the water depth $h$. As source potential in the steady problem we apply

$$
g(\mathbf{x}, \boldsymbol{\xi})=L(r, z-\zeta)+L(r, z+\zeta)
$$

where $r=\left[(x-\xi)^{2}+(y-\eta)^{2}\right]^{1 / 2}$ and

$$
L(r, z)=\left[r^{2}+z^{2}\right]^{-\frac{1}{2}}+\sum_{n=1}^{\infty}\left(\left[r^{2}+(2 n-z)^{2}\right]^{-\frac{1}{2}}+\left[r^{2}+(2 n+z)^{2}\right]^{-\frac{1}{2}}-\frac{1}{n}\right)
$$

For small and moderate $r$, the convergence of this series is slow. Thus more efficient algoritms than direct evaluation of (65) are needed. Fourier series representing $g$ can easily be obtained with the result (see Newman ${ }^{17}$, eq. 3a)

$$
L(r, z)=2 \sum_{m=0}^{\infty} \cos (m \pi z) K_{0}(m \pi r)-\gamma-\ln \frac{r}{4}
$$

Here $K_{0}$ denotes the modified Hankel function and $\gamma=0.577 \ldots$ is Euler's constant. This series converges rapidly for $r>1$. For $r \leq 1$, however, (66) converges slowly, thus a different algoritm is needed. A simple procedure is then to expand $L(r, z)$ in terms of Legendre functions. First we note that (see Abramowitz and Stegun eq. 22.9.1)

$$
\begin{aligned}
\left(r^{2}+(2 n+z)^{2}\right)^{-\frac{1}{2}} & =\frac{1}{2 n}\left(1-2 \frac{|z|}{\sqrt{z^{2}+r^{2}}} \frac{\sqrt{z^{2}+r^{2}}}{2 n}+\frac{z^{2}+r^{2}}{(2 n)^{2}}\right)^{-\frac{1}{2}} \\
& =\frac{1}{2} \sum_{q=0}^{\infty} P_{q}\left(\frac{|z|}{\sqrt{z^{2}+r^{2}}}\right)\left(\frac{z^{2}+r^{2}}{2}\right)^{\frac{q}{2}} \frac{1}{n^{q+1}}
\end{aligned}
$$

provided that

$$
\left|\frac{|z|}{n}-\frac{z^{2}+r^{2}}{(2 n)^{2}}\right|<1
$$

Here $P_{q}$ denotes the Legendre function. Applying that $P_{q}(-x)=(-1)^{q} P_{q}(x)$ and that $P_{0}(x)=1$, we obtain 


\begin{tabular}{|l|l|l|l|l|l|l|}
\hline$n$ & $m=0$ & $m=1$ & $m=2$ & $m=3$ & $m=4$ & $m=5$ \\
\hline 0 & 0.0 & -0.02525711 & 0.00086549 & -0.00004077 & 0.00000215 & -0.00000019 \\
\hline 1 & 0.05051423 & -0.00692395 & 0.00073382 & -0.00006865 & 0.00000594 & -0.00000049 \\
\hline 2 & 0.00230799 & -0.00097843 & 0.00020593 & -0.00003167 & 0.00000406 & - \\
\hline 3 & 0.00013046 & -0.00010983 & 0.00003801 & -0.00008651 & - & - \\
\hline 4 & 0.00000785 & -0.00001086 & 0.00000556 & - & - & - \\
\hline 5 & 0.00000048 & -0.00000099 & - & - & - & - \\
\hline
\end{tabular}

Table 1: Numerical values of $a_{k l}$

$$
\begin{aligned}
L(r, z) & =\left[r^{2}+z^{2}\right]^{-\frac{1}{2}}+\left[r^{2}+(2-z)^{2}\right]^{-\frac{1}{2}}+\left[r^{2}+(2+z)^{2}\right]^{-\frac{1}{2}}-1 \\
& +\sum_{q=0}^{\infty} P_{2 q}\left(\frac{|z|}{\sqrt{z^{2}+r^{2}}}\right)\left(\frac{z^{2}+r^{2}}{2}\right)^{q}(Z(2 q+1)-1)
\end{aligned}
$$

provided that $r \leq 1$. In (69) we have introduced the Zeta function $Z(q)=\sum_{n=1}^{\infty} 1 / n^{q}$. By introducing the polynomial expansions of the Legendre functions we obtain that the last sum in (69) is replaced by a power series expansion. The final result for $L$ is

$$
L(r, z)=\left[r^{2}+z^{2}\right]^{-\frac{1}{2}}+\left[r^{2}+(2-z)^{2}\right]^{-\frac{1}{2}}+\left[r^{2}+(2+z)^{2}\right]^{-\frac{1}{2}}-1+\sum_{k=0}^{\infty} \sum_{l=0}^{\infty} a_{k l} r^{2 k} z^{2 l}
$$

The coefficients $a_{k l}$ of (70) are thus obtained as products of tabulated values of the Legendre polynomial coefficients, the binominal coefficients of $\left(z^{2}+r^{2}\right)^{q}$ and the Zeta function. The first 25 coefficients are displayed in table 1. (70) was also obtained by Newman ${ }^{17}$ by means of Chebychev expansion and application of numerical integration.

\section{References}

[1] Wichers, J.E.W. ANd SluiJs, M.F. (1979), The influence of waves on the low frequency hydrodynamic coefficients of moored vessels. OTC 3625, Houston.

[2] Huijsmans, R.H.M. (1986), Wave drift forces in current. 16th Symp. on Naval Hydrodyn.

[3] Zhao, R., Faltinsen, O.M., Krokstad, J.R. and Aanesland, V. (1988), Wave-current Interaction Effects on Large-Volume Structures. Int. Conf. BOSS.

[4] ZhaO, R. AND Faltinsen, O.M. (1989), Interaction between current, waves and marine structures. 5th Int. Conf. on Num. Ship Hydrodyn., Hiroshima

[5] Nossen, J., Grue, J. ANd Palm, E. (1991), Wave forces on three-dimensional floating bodies with small forward speed. J. Fluid Mech. 227, 135-160. 
[6] GRUE, J. (1992), Drift force and drift moment on ships advancing with a small speed in oblique waves. Ship Tech. Res. 39, 22-31.

[7] Emmerhoff, O. J. ANd Sclavounos, P. D. (1992), The slow-drift motion of arrays of vertical cylinders. J. Fluid Mech. (to appear).

[8] Newman, J. N. (1992), Wave-drift damping of floating bodies. J. Fluid Mech. (to appear).

[9] Pinkster, J. A. ANd Huijsmans, R. H. M. (1992), Wave drift forces in shallow water. Proc. 16th Intnl. conference (BOSS'92), London, Great Britain. Eds. M. H. Patel and R. Gibbins.

[10] OGILvie, T. F. (1983), Second-order hydrodynamic effects on ocean platforms. Intl Workshop on Ship and Platform Motions. Univ. Calif. Berkeley.

[11] Huijsmans, R.H.M. AND Hermans, A.J. (1985), A fast algorithm for computation of 3-D ship motions at moderate forward speed. 4th International Confer. on Numerical Ship Hydrodynamics.

[12] Timman, R. AND Newman, J.N. (1962), The coupled damping coefficients of a symmetric ship. J. of Ship Res. 5, 1-7.

[13] WU, G. X. AND Eatock-TAYlor, R. (1990), The hydrodynamic force on an oscillating ship with low forward speed. J. Fluid Mech. 211, 333-353.

[14] Grue, J. AND Palm, E. (1992), The mean drift force and yaw moment on marine structures in waves and current. (Submitted)

[15] GRUe, J. AND PALM, E. (1991), Currents and wave forces on ships and marine structures, in: Dynamics of marine vehicles and structures in waves. IUTAM Symp. Brunel University, Uxbridge, 1990, Ed. W. G. Price, Elsevier.

[16] GrUe, J. AND PALM, E. (1992), The mean yaw moment on floating bodies advancing with a forward speed in waves. Proc. 16th Intnl. conference (BOSS'92), London, Great Britain. Eds. M. H. Patel and R. Gibbins.

[17] Newman, J.N. (1991), The approximation of free-surface Green functions, in: Wave asymptotics - Proc. of the Fritz Ursell Retirement Meeting, Camb. Univ. Press. 
Figure captions

Figure 1.

Solid line: $C_{g}(K h)=\tanh K h+\frac{K h}{\cosh ^{2} K h}$. Dotted line: $\tanh K h$

Figure 2.

$a_{13}(U)$ (solid line) and $a_{31}(-U)$ (dotted line) for a half-immersed sphere vs. $\sigma^{2} a / g$. $F r=$ 0.04 . a) $h=\infty$ b) $h / a=2$ c) $h / a=1.2$. 392 panels on half- $S_{B}, 896$ panels on half- $S_{F}$. Free surface discretized out to a radius of $6 a$.

Figure 3.

Surge exciting force $X_{1}$ for a half-immersed sphere vs. wave number. $\beta=\pi / 2, F r=0.04$. Solid line: Generalized Haskind relation $(h / a=1.2)$. Dashed line: Pressure integration $(h / a=1.2)$. Dotted line: Generalized Haskind relation $(h=\infty)$. Same discretization as in figure 2.

Figure 4.

a) Surge exciting force $X_{1}$ and b) heave exciting force $X_{3}$ obtained by the generalized Haskind relations for a half-immersed sphere vs. wave number. $\beta=\pi$. Solid line: $F r=$ 0.04, $h / a=1.2$. Dashed line: $F r=-0.04, h / a=1.2$. Dense dotted line: $F r=0.04$, $h=\infty$. Sparce dotted line: $F r=-0.04, h=\infty$. Same discretization as in figure 2 .

Figure 5

Same as figure 4, but a) $\partial X_{1} / \partial F r$ and b) $\partial X_{3} / \partial F r$.

Figure 6.

Mean drift force $F_{x}$ for a half-immersed sphere vs. wave number. $\beta=\pi$. a) Solid line: $F r=0.04, h / a=2$. Dashed line: $F r=-0.04, h / a=2$. Dotted lines: Corresponding values for $h=\infty$. b) $h / a=1$.2. Solid line: $F r=0.04$, Dashed line: $F r=-0.04$. c) Wave drift damping coefficient $F_{x 1}$ for the half-immersed sphere vs. wave number. $\beta=\pi$, Solid line: $h / a=1.2$. Dashed line: $h / a=2$. Dotted line: $h=\infty$.

Figure 7.

a) Mean drift force at zero speed, $F_{x 0}$, and b) wave drift damping coefficient, $F_{x 1}$, for a vertical truncated circular cylinder with radius $a$ and draught $a$, vs. wave number. $\beta=\pi$. Solid line: $h / a=1$ Dotted line: $h=\infty$. 330 panels on half- $S_{B}, 957$ panels on half- $S_{F}$. Free surface discretized out to a radius of $4 a$. 
Figure 8.

a) Mean drift force at zero speed, $F_{x 0}$, and b) wave drift damping coefficient, $F_{x 1}$, for a ship (length $L=230 \mathrm{~m}$, beam $B=41 \mathrm{~m}$ and draught $T=15 \mathrm{~m}$ ) vs. wave number. $\beta=\pi .380$ panels on half- $S_{B}, 900$ panels on half- $S_{F}$. Solid line (squares mark computation points): $h=60 m$. Dotted line: $h=\infty$.

Figure 9.

Same as figure 8 , but $h=30 m$.

Figure 10.

Same as figure 8 , but $h=18 m$.

Figure 11.

$a_{13}(U)$ (squares) and $a_{31}(-U)$ (triangles) for a restrained half-immersed sphere vs. $1 / N_{B}$. $N_{F} \simeq 2.2 \times N_{B} . F r=0.04, h / a=1.2, \sigma^{2} a / g=0.8$.

Figure 12

Mean drift force, $F_{x}$, and wave drift damping coefficient, $F_{x 1}$, for a restrained half-immersed sphere vs. $1 / N_{B} . N_{F} \simeq 2.2 \times N_{B} . h / a=1.2, K a=1.1391, \beta=\pi$. a) $F_{x}$ at $F r=0$. b) $F_{x}$ at $F r=0.04$. c) Wave drift damping coefficient $F_{x 1}$.

Figure 13

Energy flux at $S_{\infty}$ due to a restrained half-immersed sphere vs. Froude number. $W / 2 a E c_{g}$ (squares) and $W^{\prime} / 2 a E c_{g}$ (triangles). $h / a=1.2, K a=1.1391$ and $\beta=\pi$. Same discretization as in figure 2. 


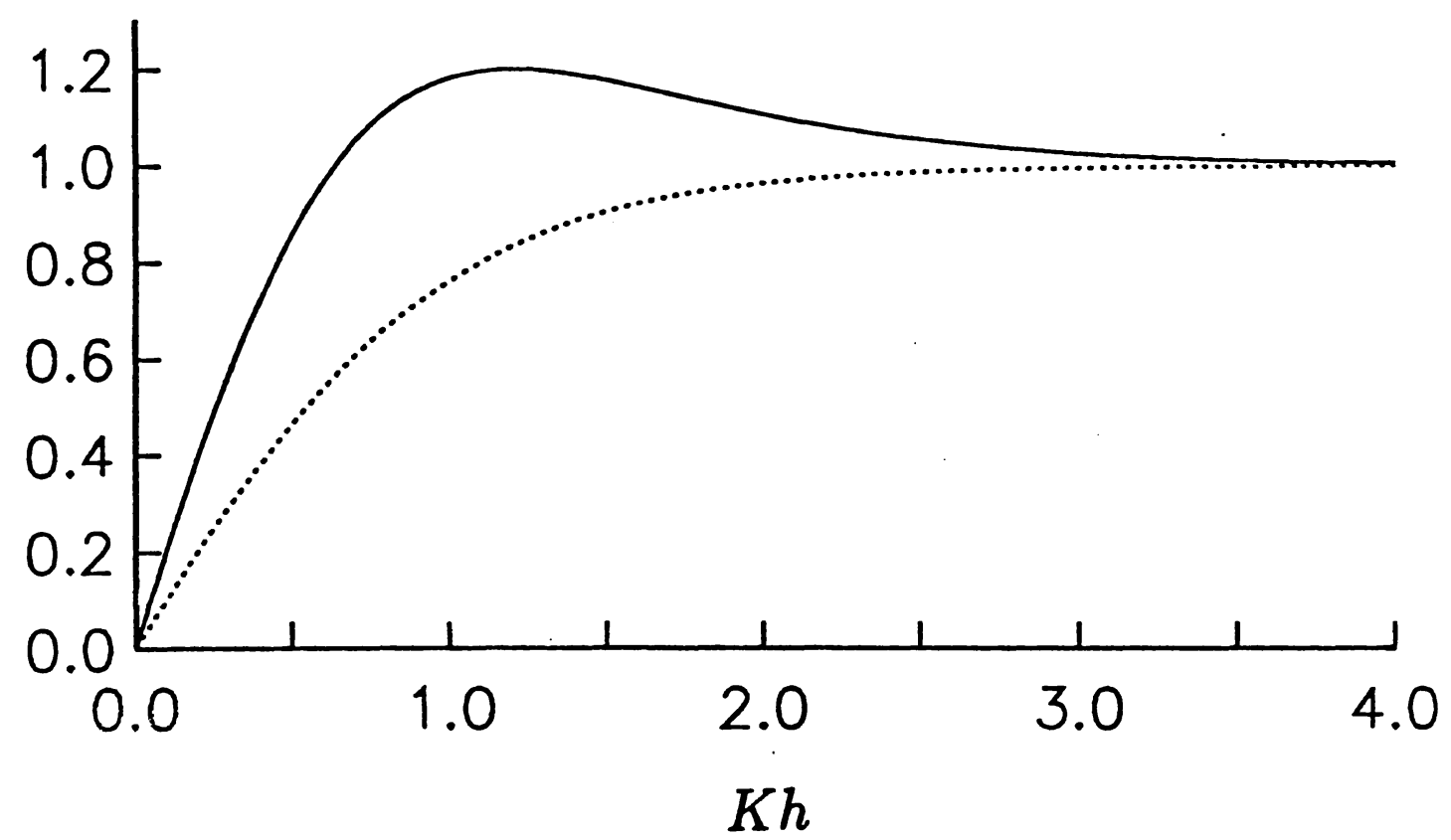

Figure 1.

Solid line: $C_{g}(K h)=\tanh K h+\frac{K h}{\cosh ^{2} K h}$. Dotted line: $\tanh K h$ 
a)

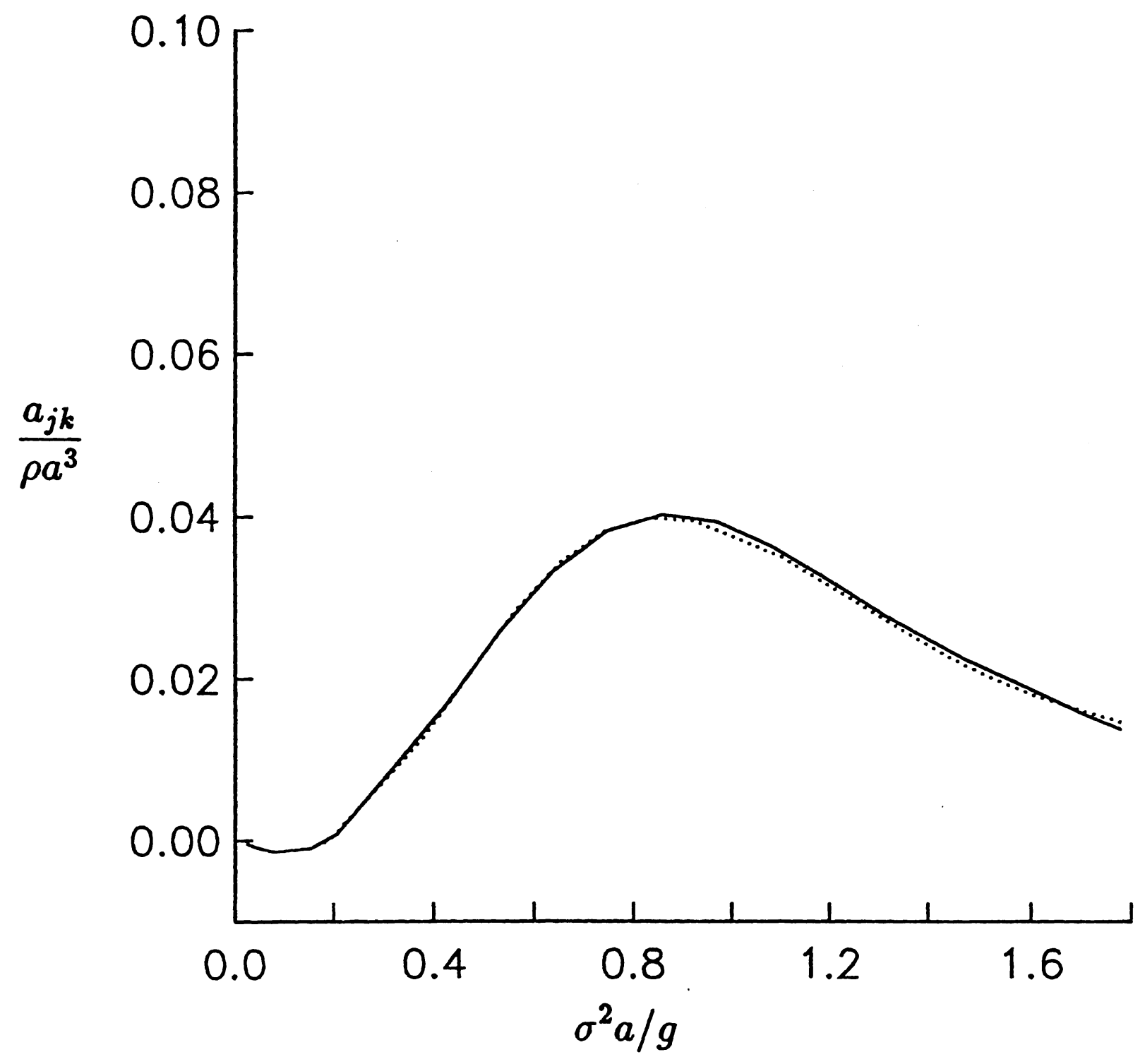

Figure 2.

$a_{13}(U)$ (solid line) and $a_{31}(-U)$ (dotted line) for a half-immersed sphere vs. $\sigma^{2} a / g . F r=$ 0.04 . a) $h=\infty$ b) $h / a=2$ c) $h / a=1.2$. 392 panels on half- $S_{B}, 896$ panels on half- $S_{F}$. Free surface discretized out to a radius of $6 a$. 
b)

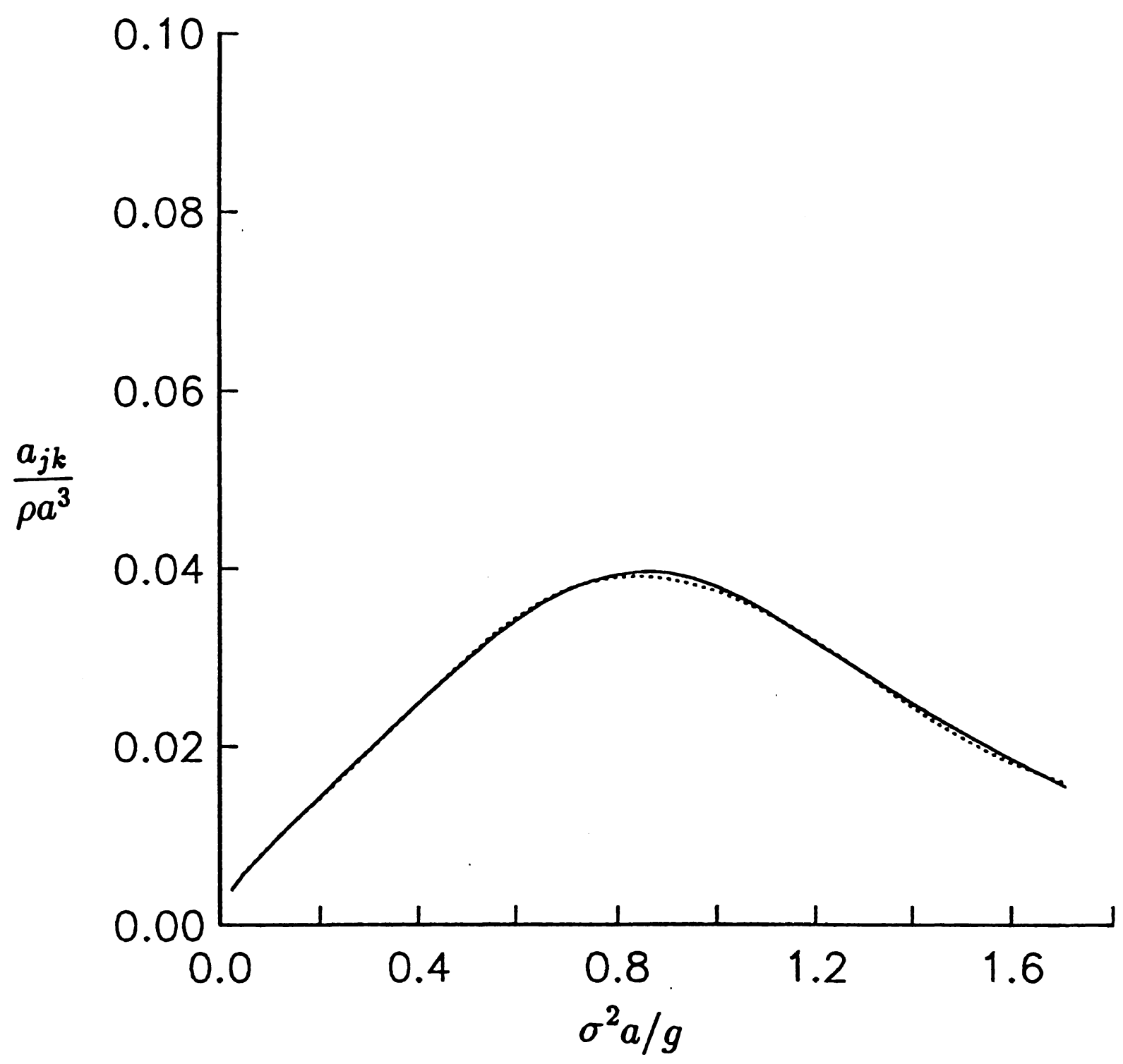

Figure 2.

$a_{13}(U)$ (solid line) and $a_{31}(-U)$ (dotted line) for a half-immersed sphere vs. $\sigma^{2} a / g . F r=$ 0.04. a) $h=\infty$ b) $h / a=2$ c) $h / a=1.2$. 392 panels on half- $S_{B}, 896$ panels on half- $S_{P}$. Free surface discretized out to a radius of $6 a$. 
c)

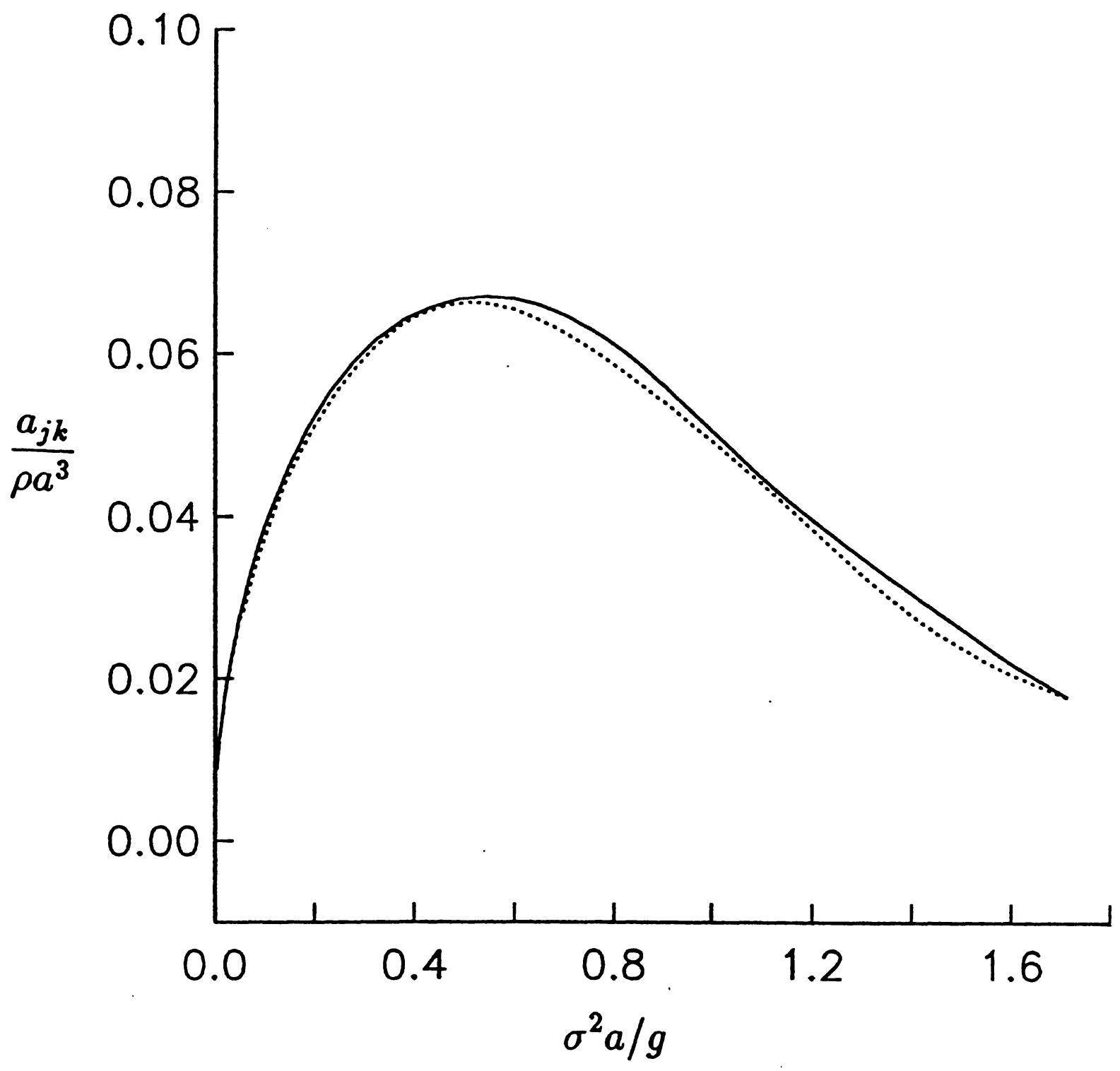

Figure 2.

$a_{13}(U)$ (solid line) and $a_{31}(-U)$ (dotted line) for a half-immersed sphere vs. $\sigma^{2} a / g . F r=$ 0.04 . a) $h=\infty$ b) $h / a=2$ c) $h / a=1.2$. 392 panels on half- $S_{B}, 896$ panels on half- $S_{F}$. Free surface discretized out to a radius of $6 a$. 


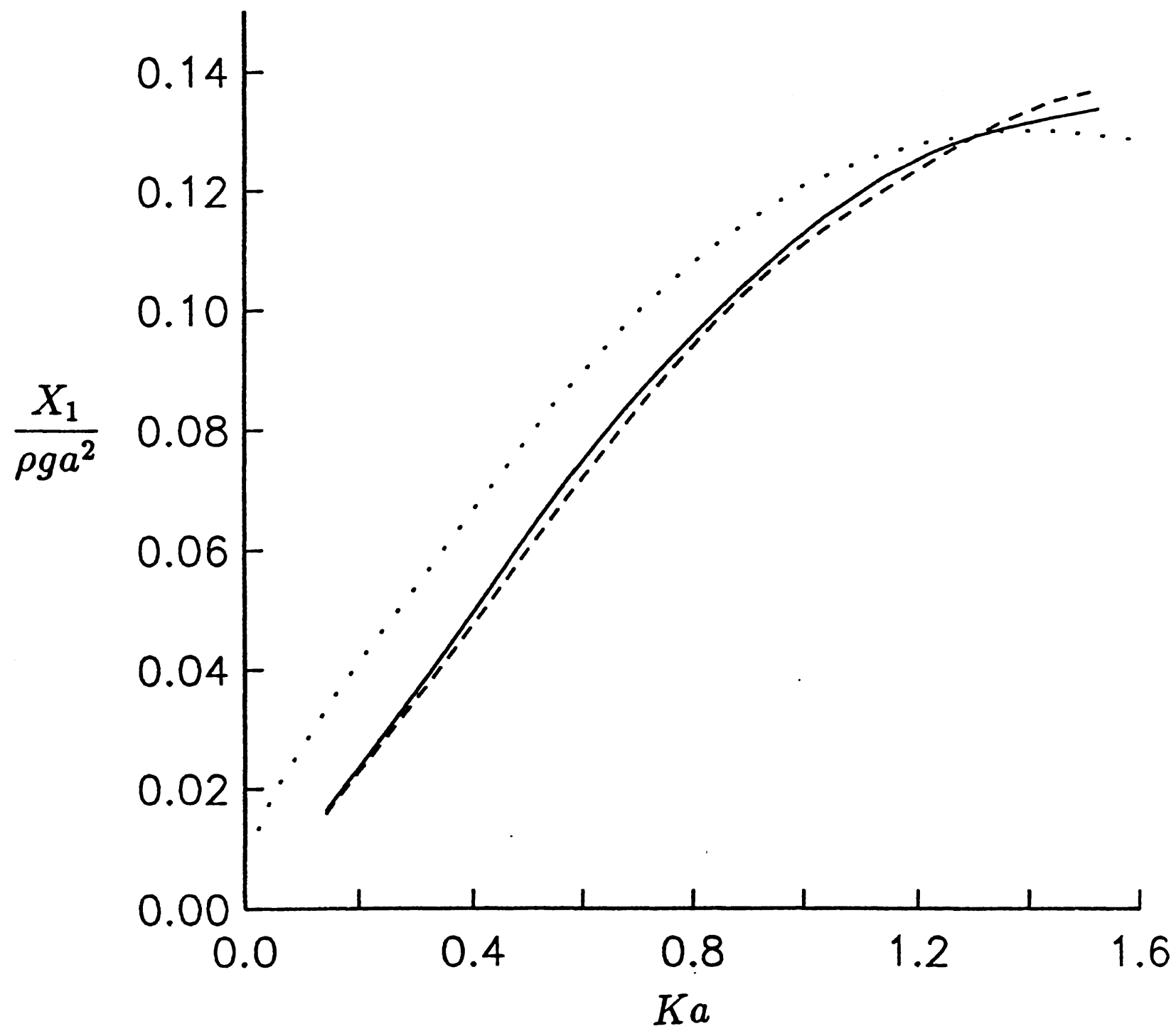

Figure 3.

Surge exciting force $X_{1}$ for a half-immersed sphere vs. wave number. $\beta=\pi / 2, F r=0.04$. Solid line: Generalized Haskind relation $(h / a=1.2)$. Dashed line: Pressure integration $(h / a=1.2)$. Dotted line: Generalized Haskind relation $(h=\infty)$. Same discretization as in figure 2. 
a)

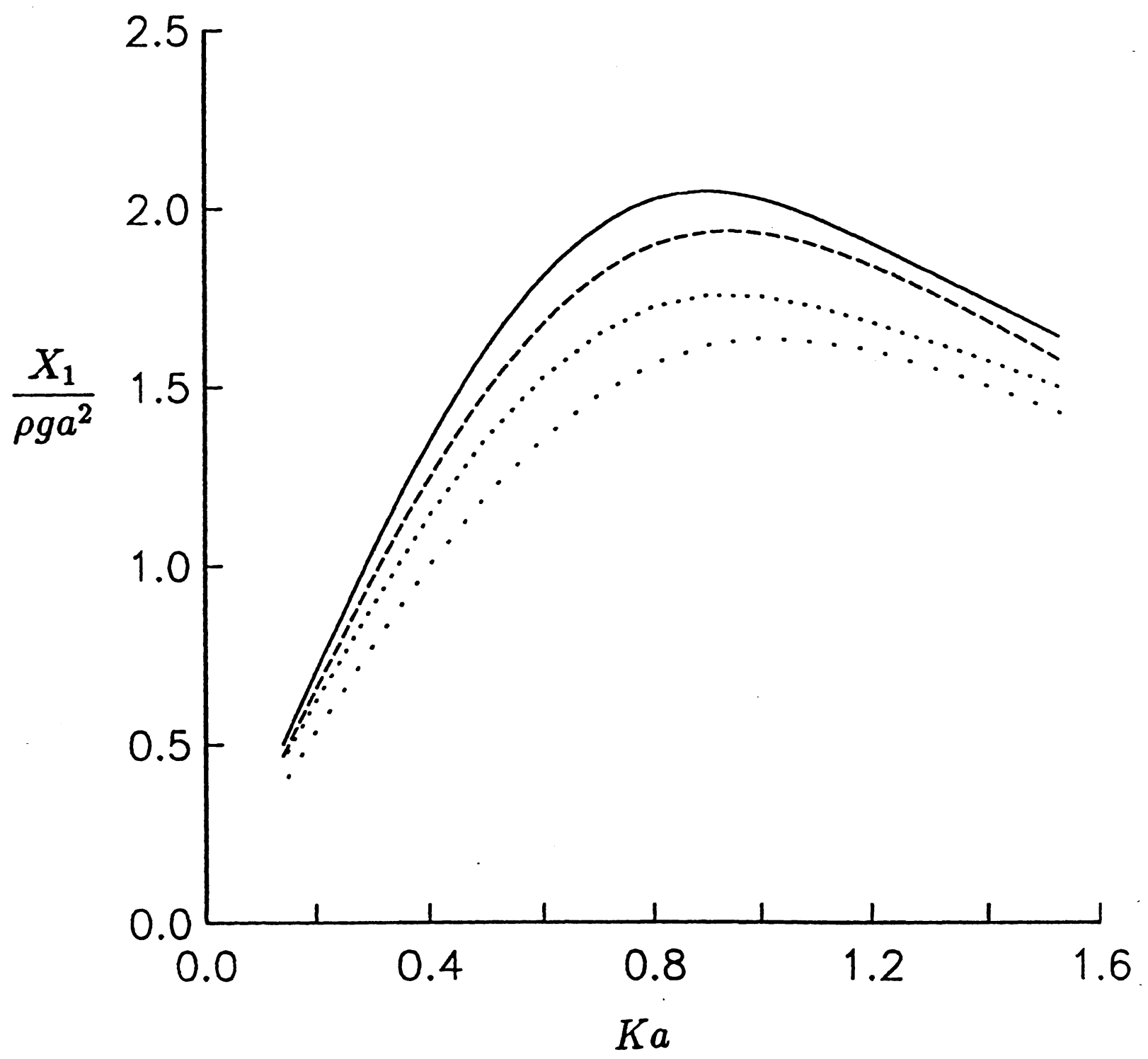

Figure 4.

a) Surge exciting force $X_{1}$ and b) heave exciting force $X_{3}$ obtained by the generalized Haskind relations for a half-immersed sphere vs. wave number. $\beta=\pi$. Solid line: $F r=$ 0.04, $h / a=1.2$. Dashed line: $F r=-0.04, h / a=1.2$. Dense dotted line: $F r=0.04$, $h=\infty$. Sparce dotted line: $F_{r}=-0.04, h=\infty$. Same discretization as in figure 2. 
b)

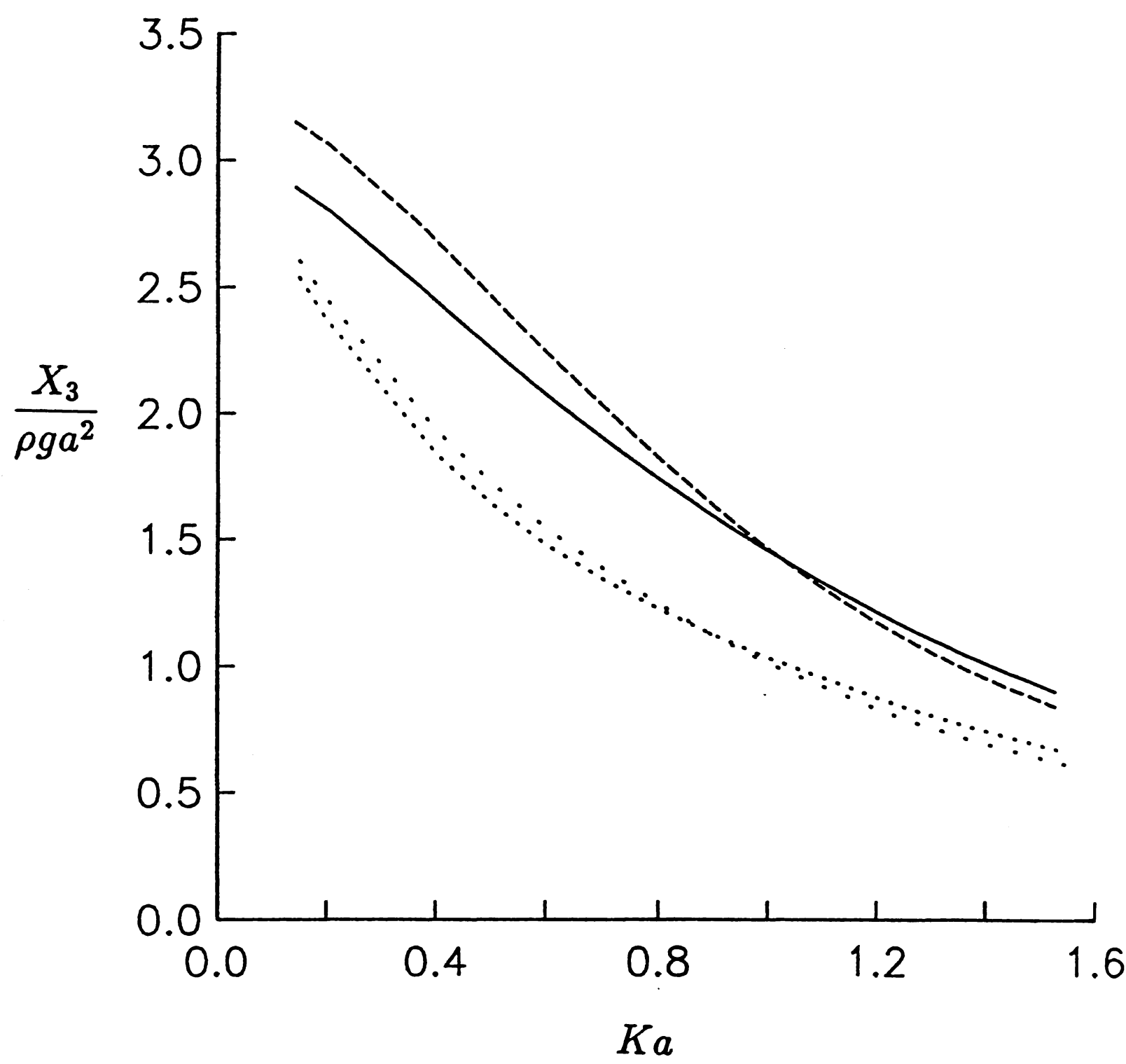

Figure 4.

a) Surge exciting force $X_{1}$ and b) heave exciting force $X_{3}$ obtained by the generalized Haskind relations for a half-immersed sphere vs. wave number. $\beta=\pi$. Solid line: $F r=$ 0.04, $h / a=1.2$. Dashed line: $F r=-0.04, h / a=1.2$. Dense dotted line: $F r=0.04$, $h=\infty$. Sparce dotted line: $F r=-0.04, h=\infty$. Same discretization as in figure 2 . 
a)

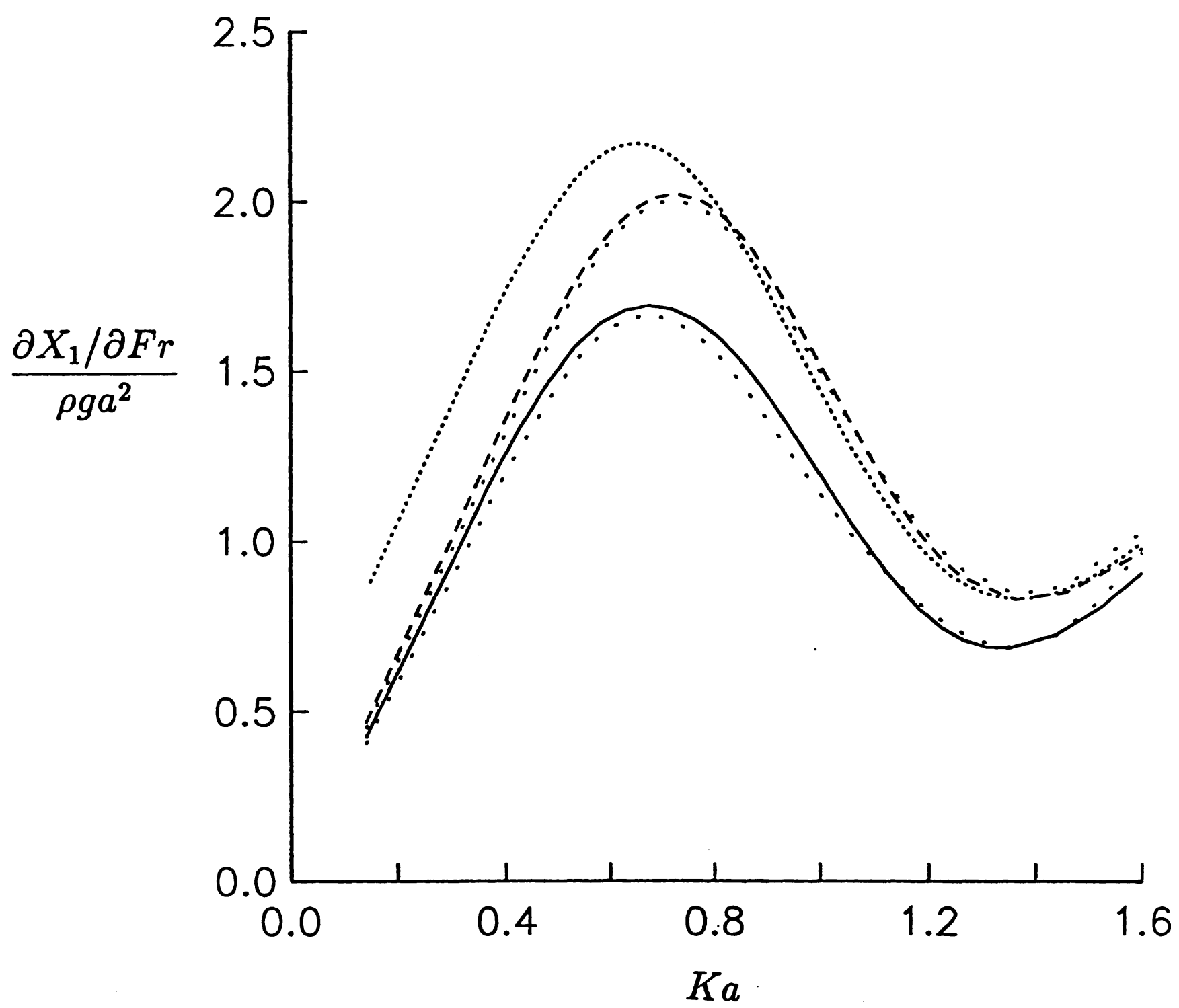

Figure 5

Same as figure 4 , but a) $\partial X_{1} / \partial F r$ and b) $\partial X_{3} / \partial F r$. 
b)

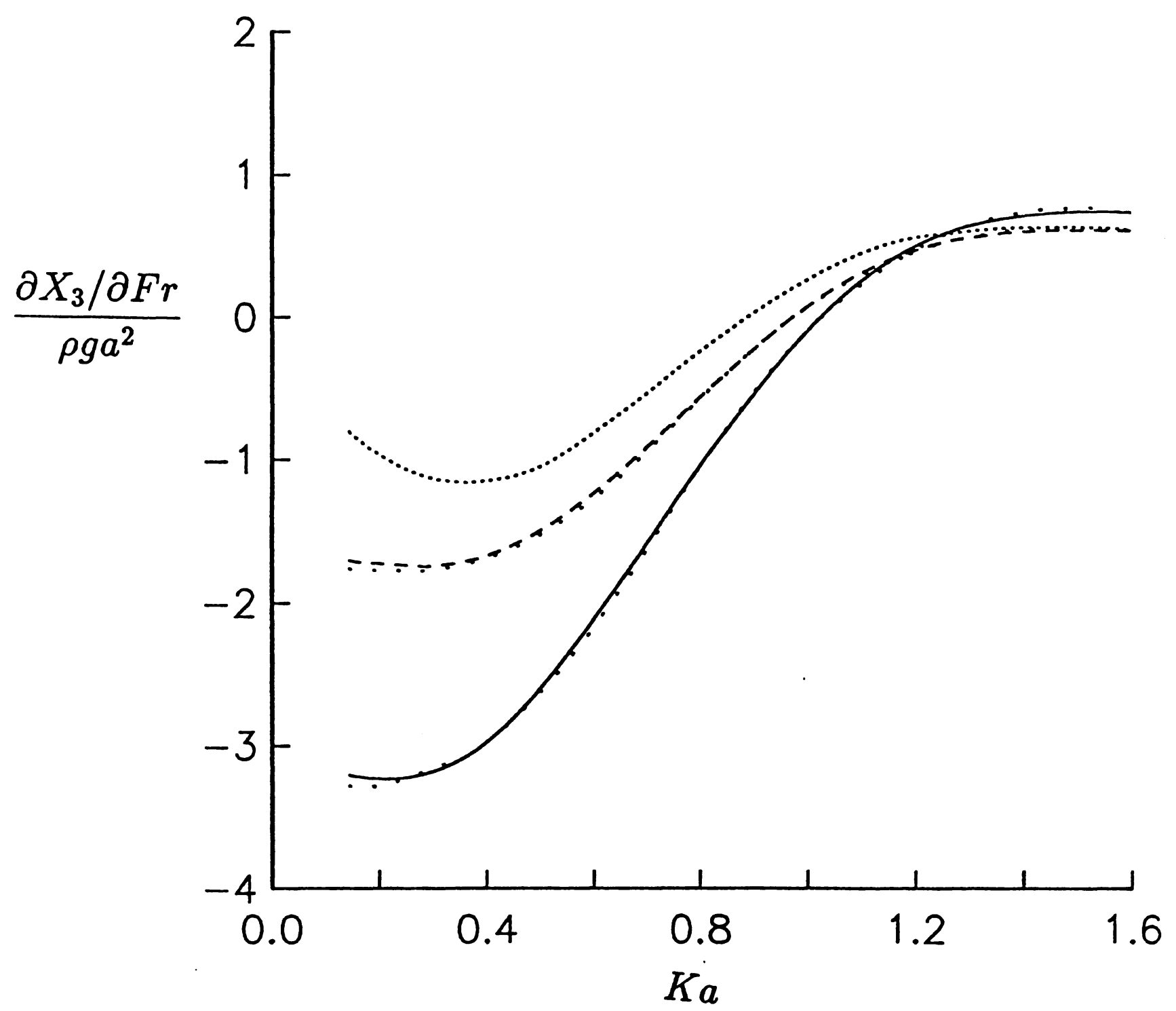

Figure 5

Same as figure 4, but a) $\partial X_{1} / \partial F r$ and b) $\partial X_{3} / \partial F r$. 
a)

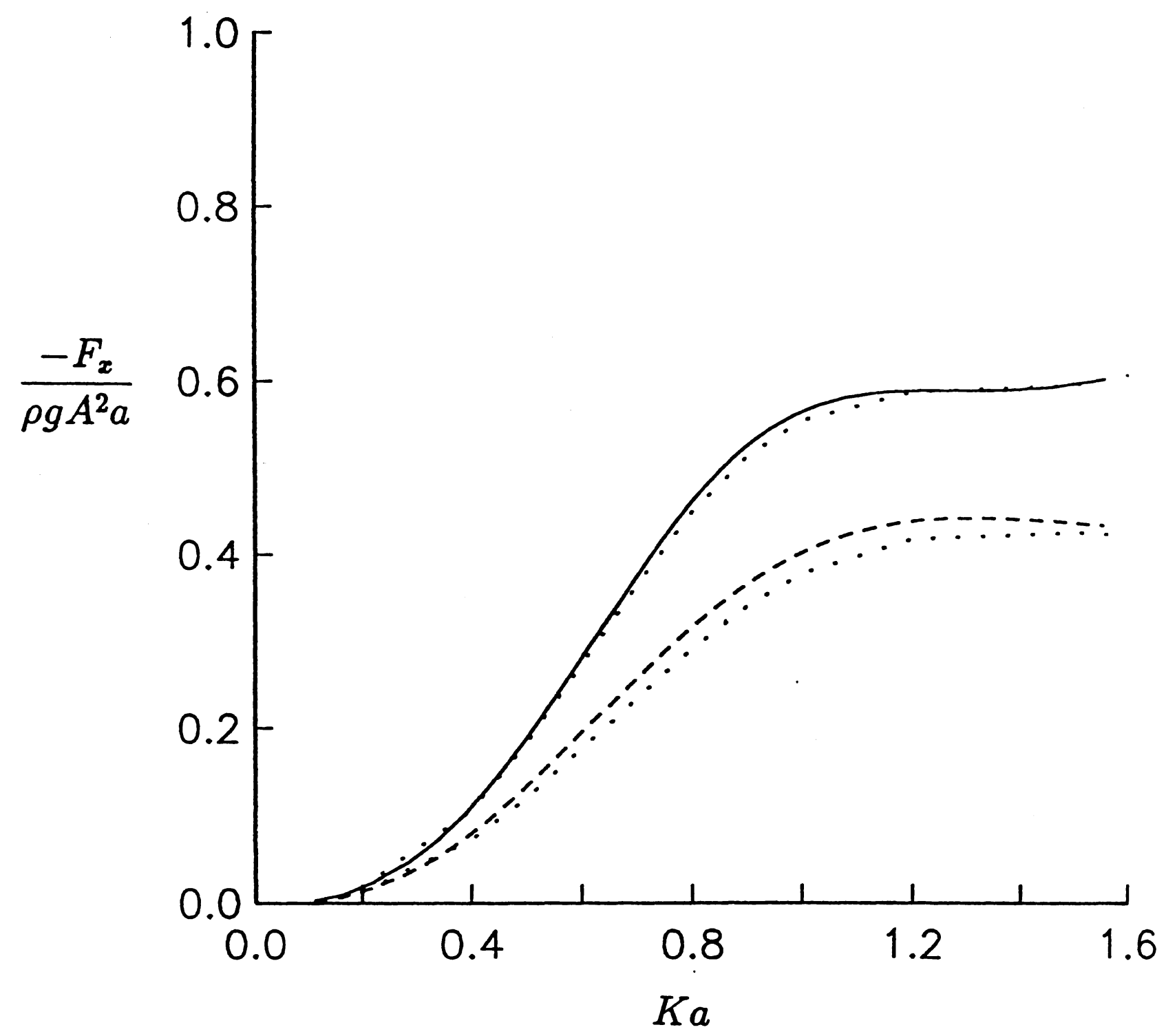

Figure 6.

Mean drift force $F_{x}$ for a half-immersed sphere vs. wave number. $\beta=\pi$. a) Solid line: $F r=0.04, h / a=2$. Dashed line: $F r=-0.04, h / a=2$. Dotted lines: Corresponding values for $h=\infty$. b) $h / a=1.2$. Solid line: $F r=0.04$, Dashed line: $F r=-0.04$. c) Wave drift damping coefficient $F_{x 1}$ for the half-immersed sphere vs. wave number. $\beta=\pi$, Solid line: $h / a=1.2$. Dashed line: $h / a=2$. Dotted line: $h=\infty$. 
b)

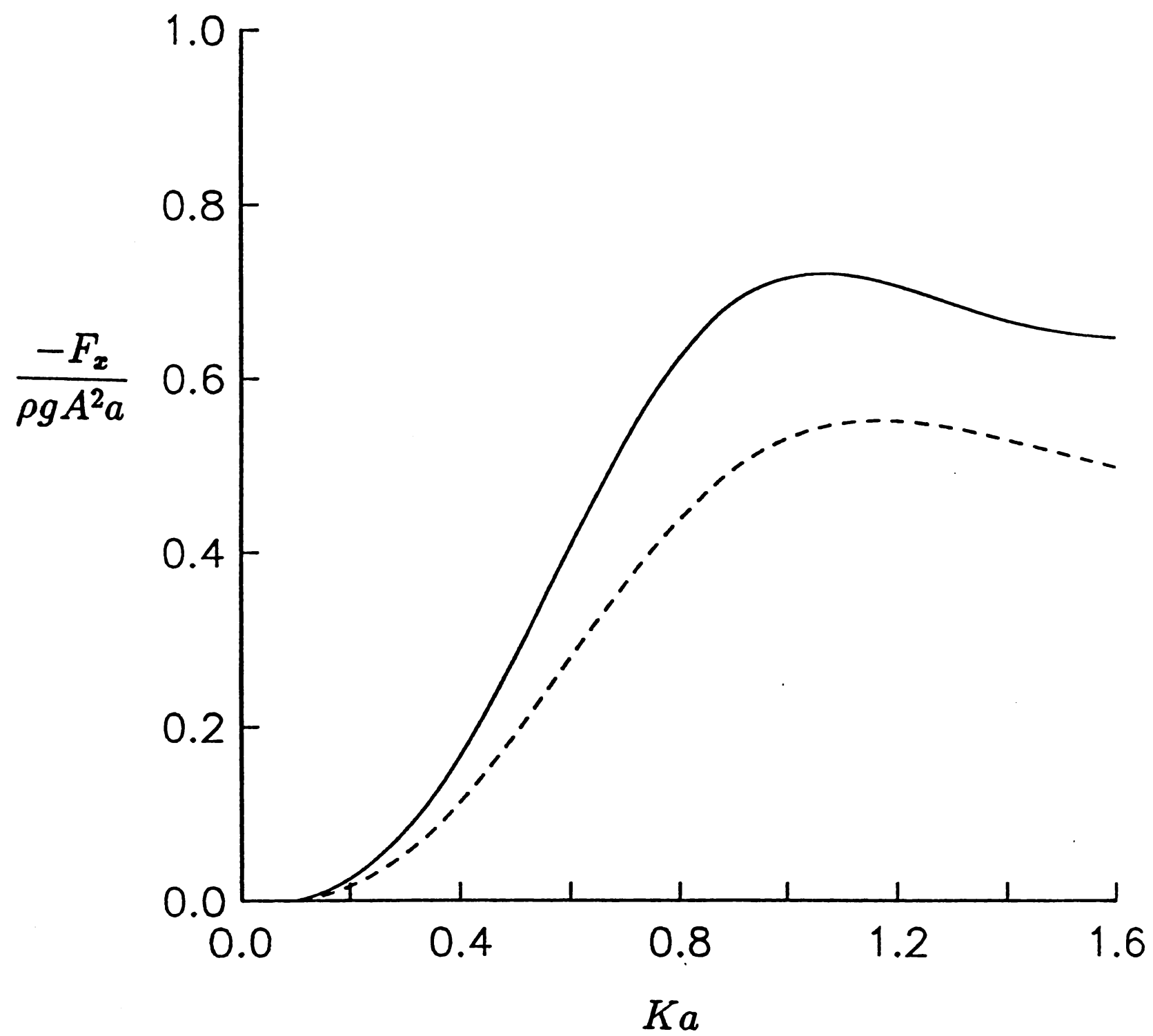

Figure 6.

Mean drift force $F_{x}$ for a half-immersed sphere vs. wave number. $\beta=\pi$. a) Solid line: $F r=0.04, h / a=2$. Dashed line: $F r=-0.04, h / a=2$. Dotted lines: Corresponding values for $h=\infty$. b) $h / a=1.2$. Solid line: $F r=0.04$, Dashed line: $F r=-0.04$. c) Wave drift damping coefficient $F_{x 1}$ for the half-immersed sphere vs. wave number. $\beta=\pi$, Solid line: $h / a=1.2$. Dashed line: $h / a=2$. Dotted line: $h=\infty$. 
c)

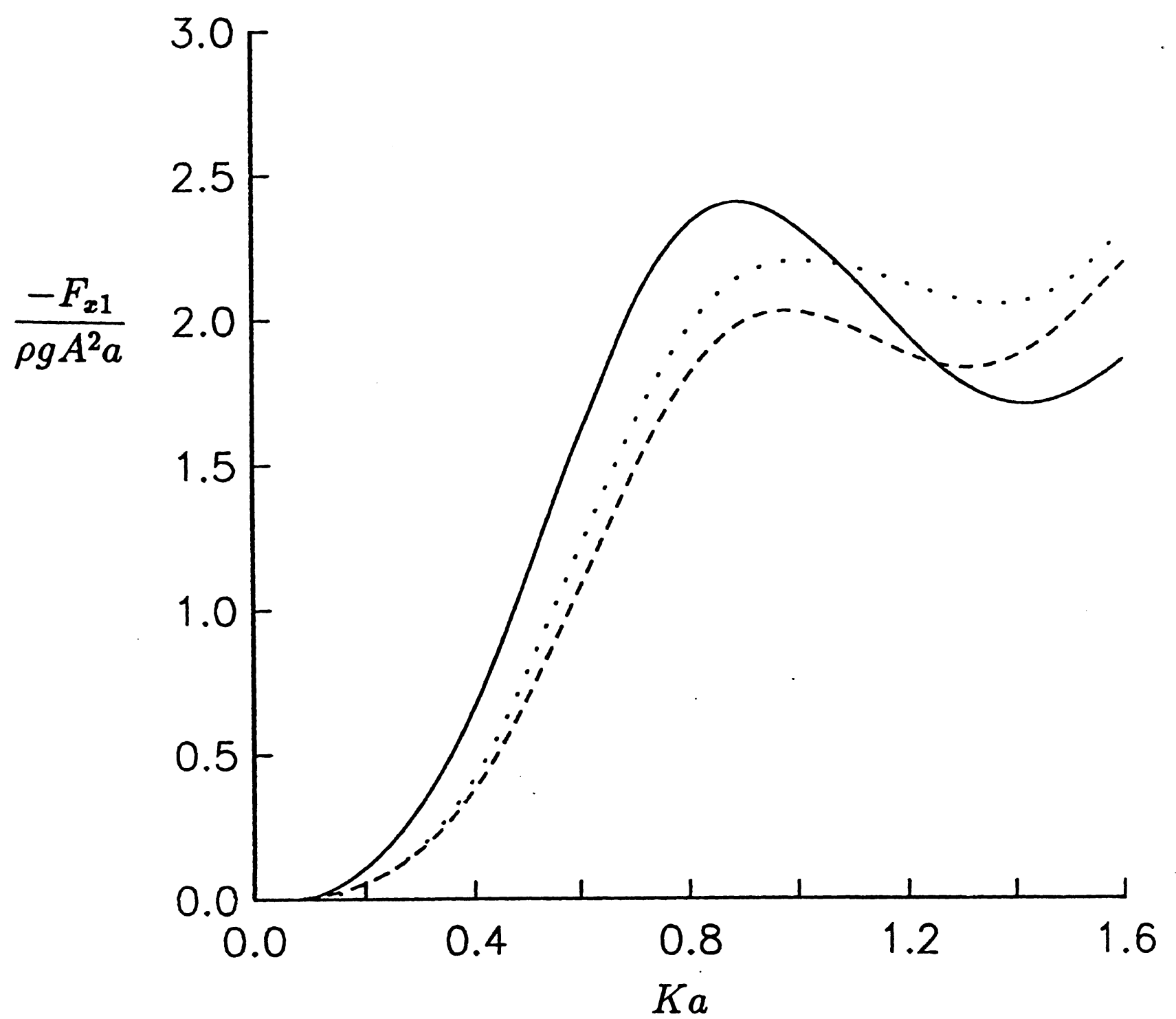

Figure 6.

Mean drift force $F_{x}$ for a half-immersed sphere vs. wave number. $\beta=\pi$. a) Solid line: $F r=0.04, h / a=2$. Dashed line: $F r=-0.04, h / a=2$. Dotted lines: Corresponding values for $h=\infty$. b) $h / a=1.2$. Solid line: $F r=0.04$, Dashed line: $F r=-0.04$. c) Wave drift damping coefficient $F_{x 1}$ for the half-immersed sphere vs. wave number. $\beta=\pi$, Solid line: $h / a=1.2$. Dashed line: $h / a=2$. Dotted line: $h=\infty$. 
a)

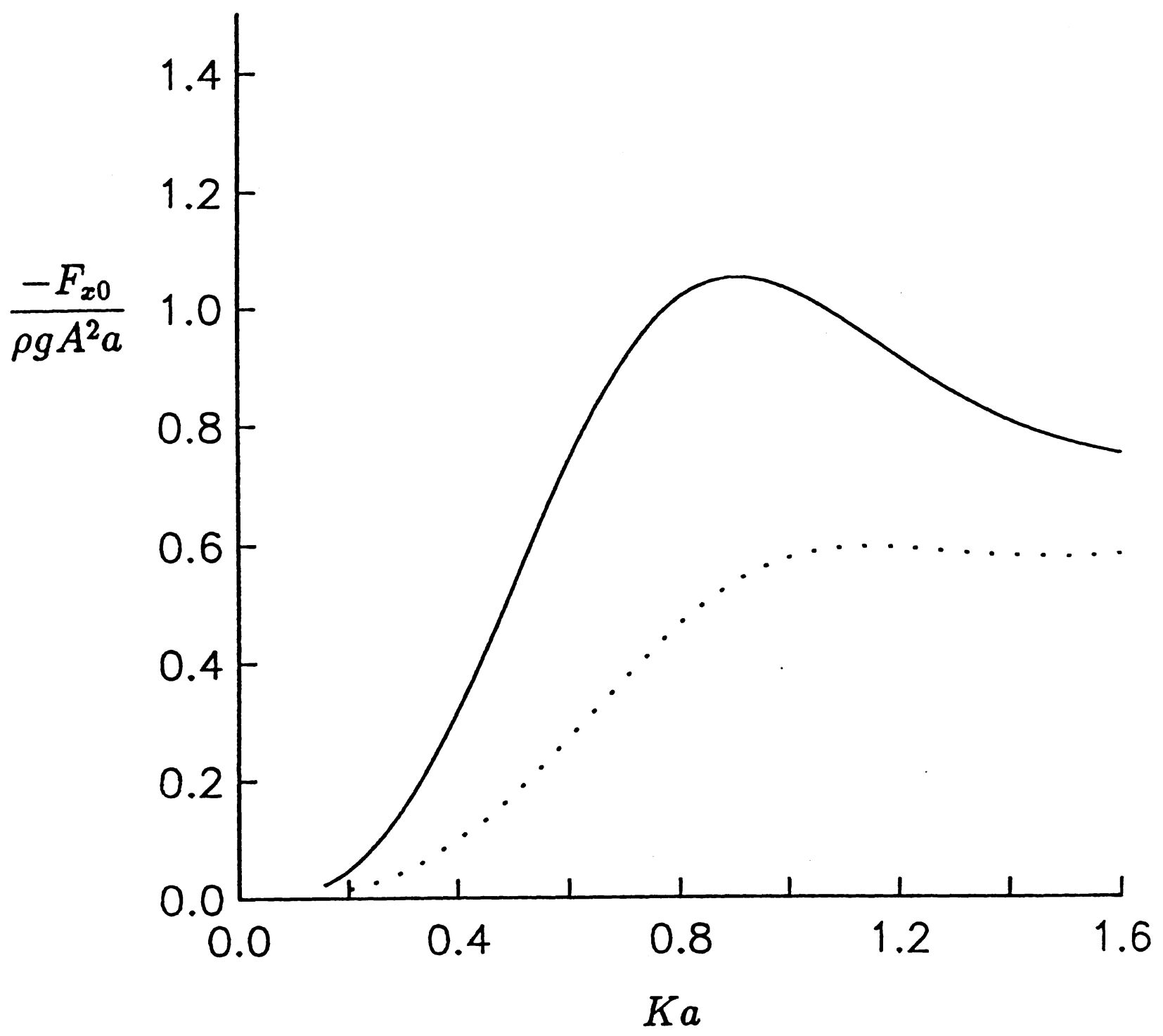

Figure 7.

a) Mean drift force at zero speed, $F_{x 0}$, and b) wave drift damping coefficient, $F_{x 1}$, for a vertical truncated circular cylinder with radius $a$ and draught $a$, vs. wave number. $\beta=\pi$. Solid line: $h / a=1$ Dotted line: $h=\infty$. 330 panels on half $S_{B}, 957$ panels on half- $S_{F}$. Free surface discretized out to a radius of $4 a$. 
b)

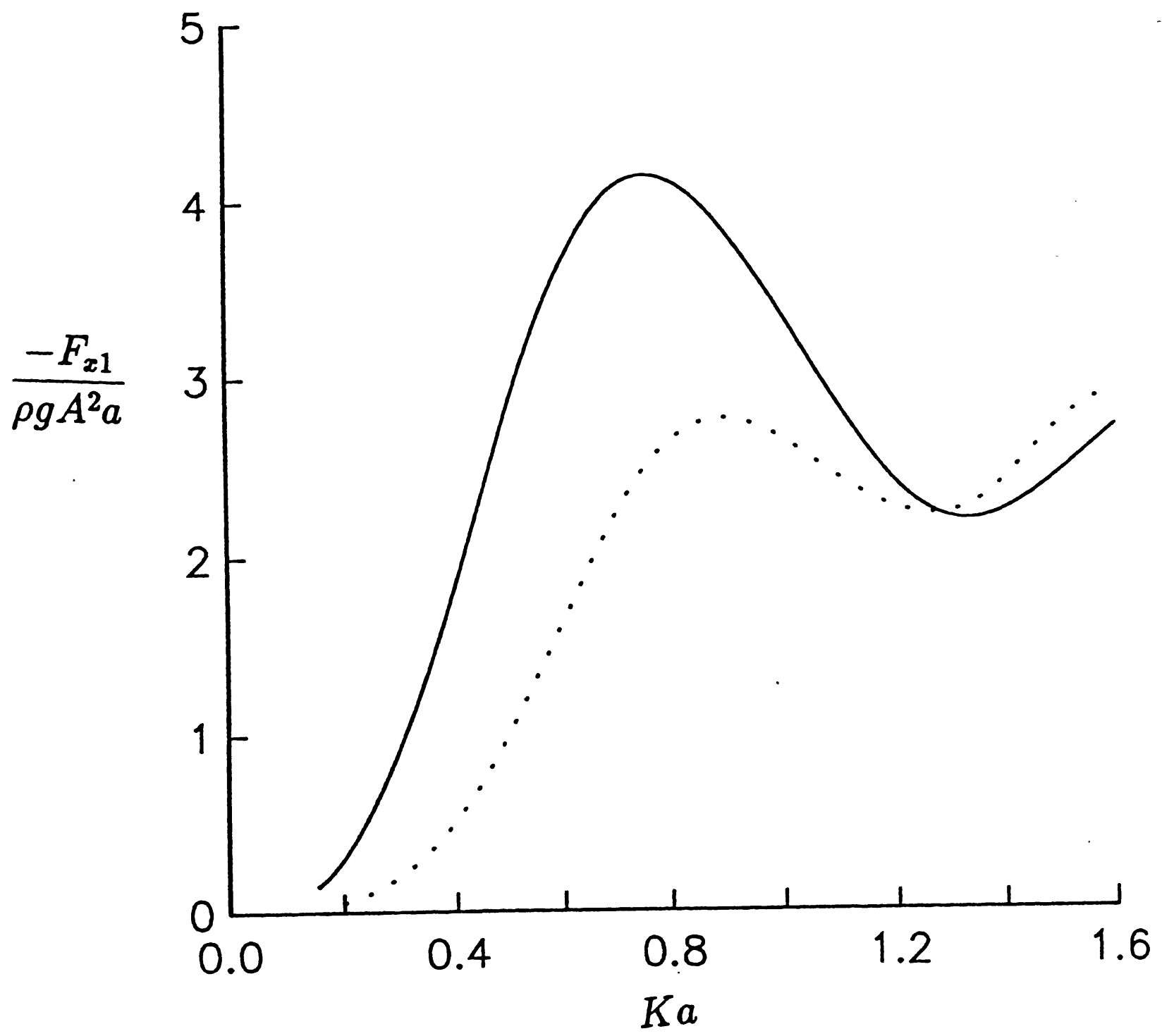

Figure 7.

a) Mean drift force at zero speed, $F_{x 0}$, and b) wave drift damping coefficient, $F_{x 1}$, for a vertical truncated circular cylinder with radius $a$ and draught $a$, vs. wave number. $\beta=\pi$. Solid line: $h / a=1$ Dotted line: $h=\infty$. 330 panels on half- $S_{B}, 957$ panels on half- $S_{F}$. Free surface discretized out to a radius of $4 a$. 
a)

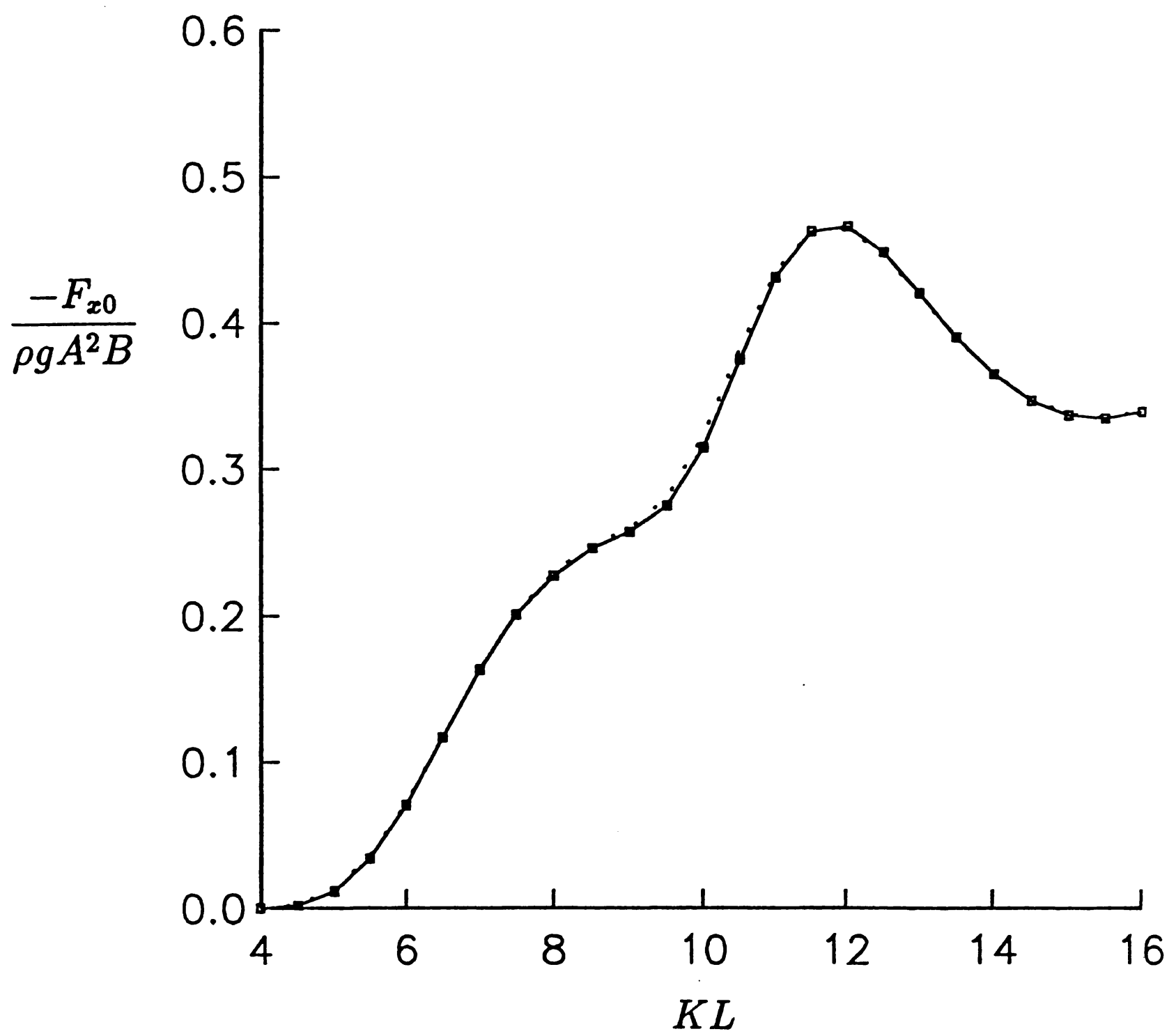

Figure 8.

a) Mean drift force at zero speed, $F_{x 0}$, and b) wave drift damping coefficient, $F_{x 1}$, for a ship (length $L=230 \mathrm{~m}$, beam $B=41 \mathrm{~m}$ and draught $T=15 \mathrm{~m}$ ) vs. wave number. $\beta=\pi .380$ panels on half- $S_{B}, 900$ panels on half- $S_{F}$. Solid line (squares mark computation points): $h=60 \mathrm{~m}$. Dotted line: $h=\infty$. 
b)

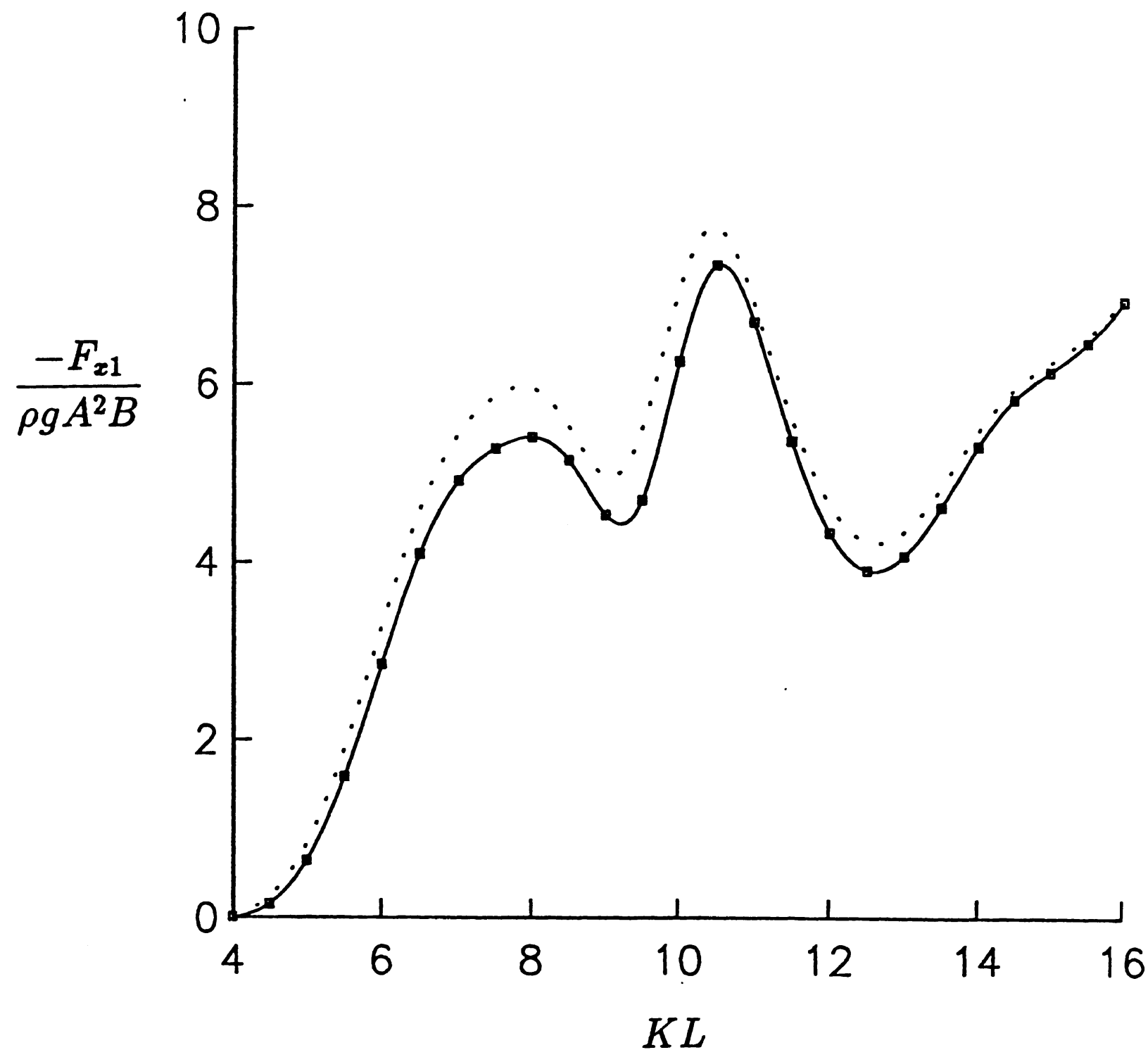

Figure 8.

a) Mean drift force at zero speed, $F_{x 0}$, and b) wave drift damping coefficient, $F_{x 1}$, for a ship (length $L=230 \mathrm{~m}$, beam $B=41 \mathrm{~m}$ and draught $T=15 \mathrm{~m}$ ) vs. wave number. $\beta=\pi$. 380 panels on half- $S_{B}, 900$ panels on half- $S_{F}$. Solid line (squares mark computation points): $h=60 m$. Dotted line: $h=\infty$. 
a)

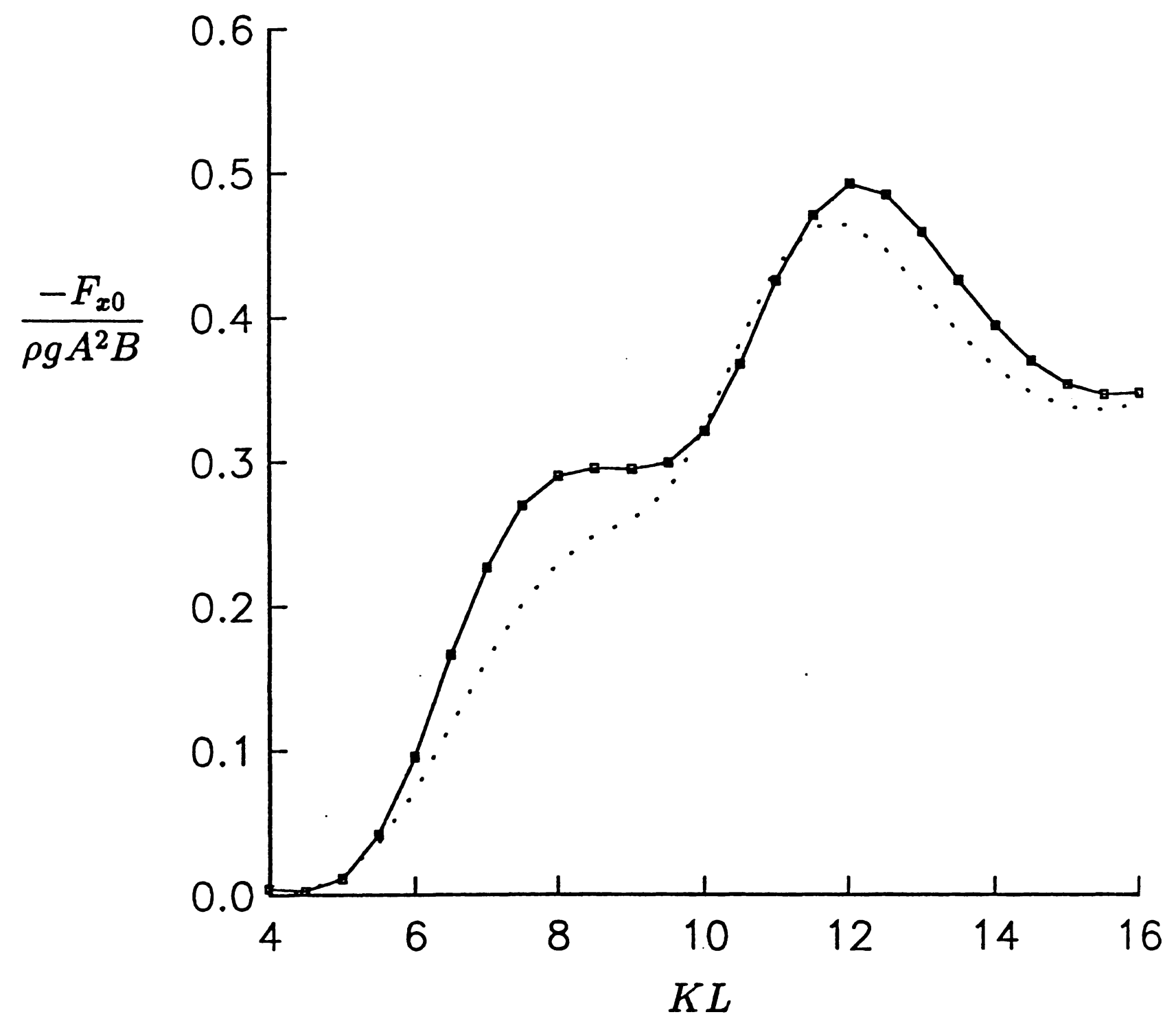

Figure 9.

Same as figure 8 , but $h=30 m$. 
b)

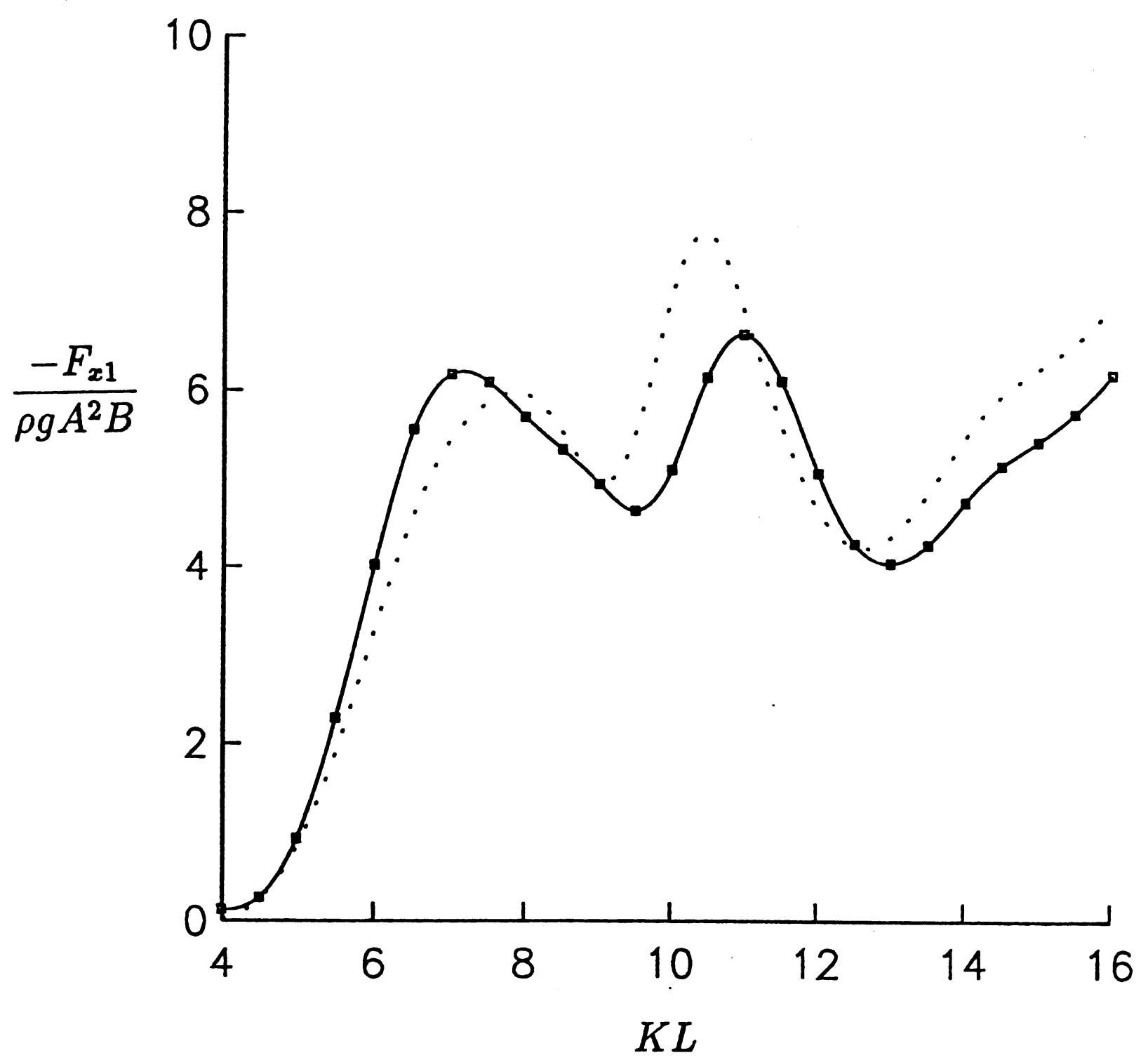

Figure 9.

Same as figure 8 , but $h=30 \mathrm{~m}$. 
a)

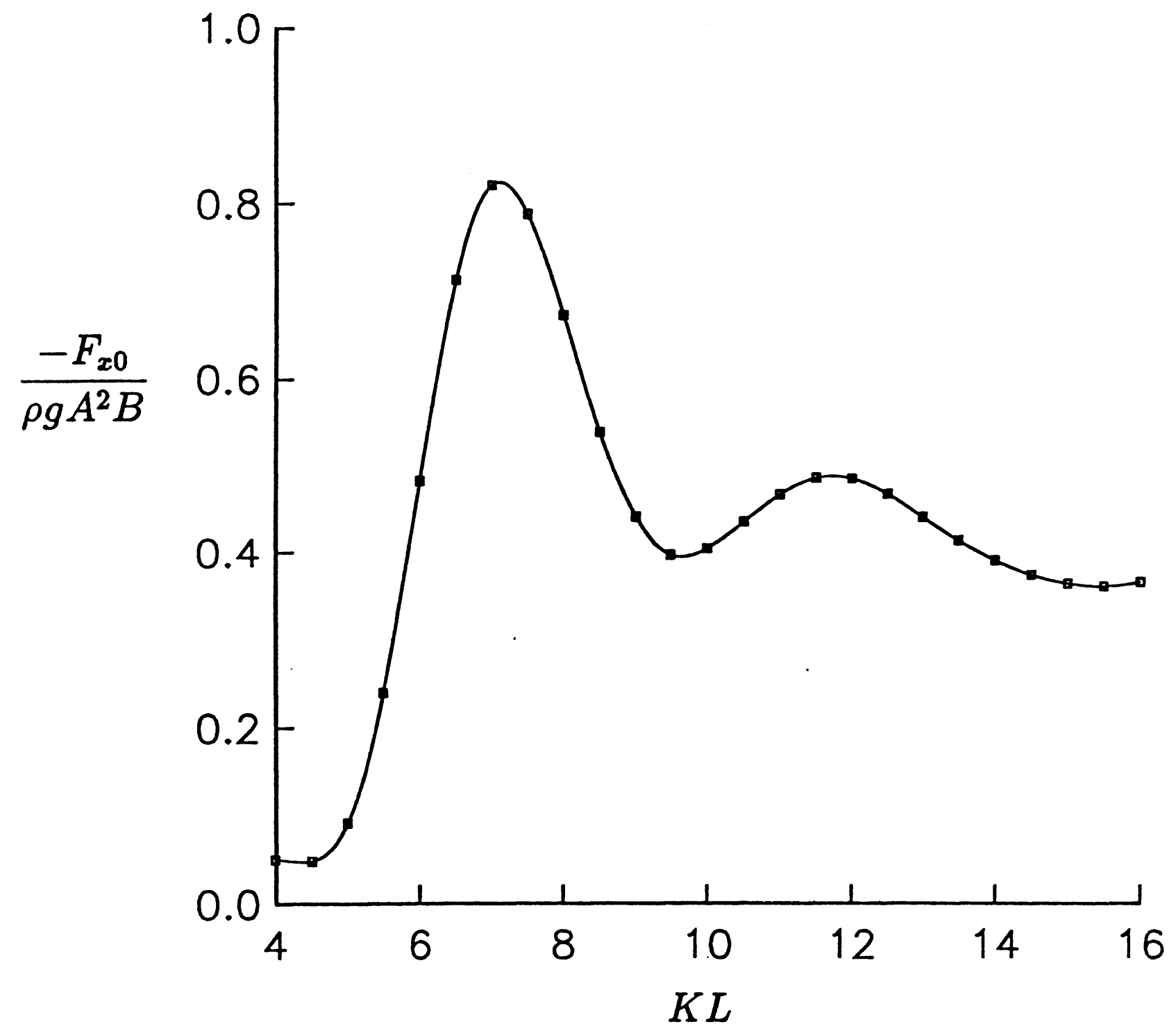

Figure 10.

Same as figure 8 , but $h=18 m$. 
b)

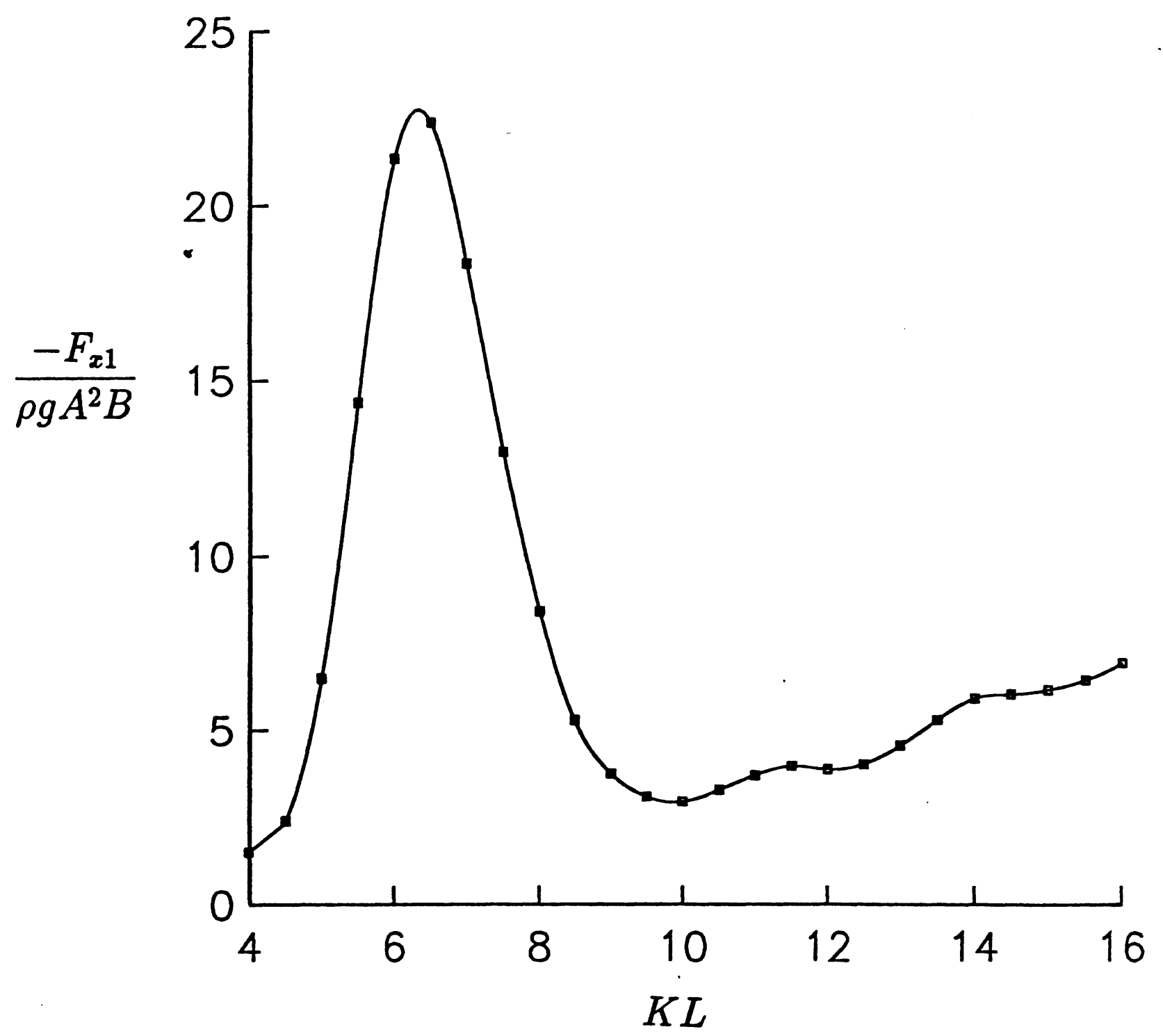

Figure 10.

Same as figure 8 , but $h=18 m$. 


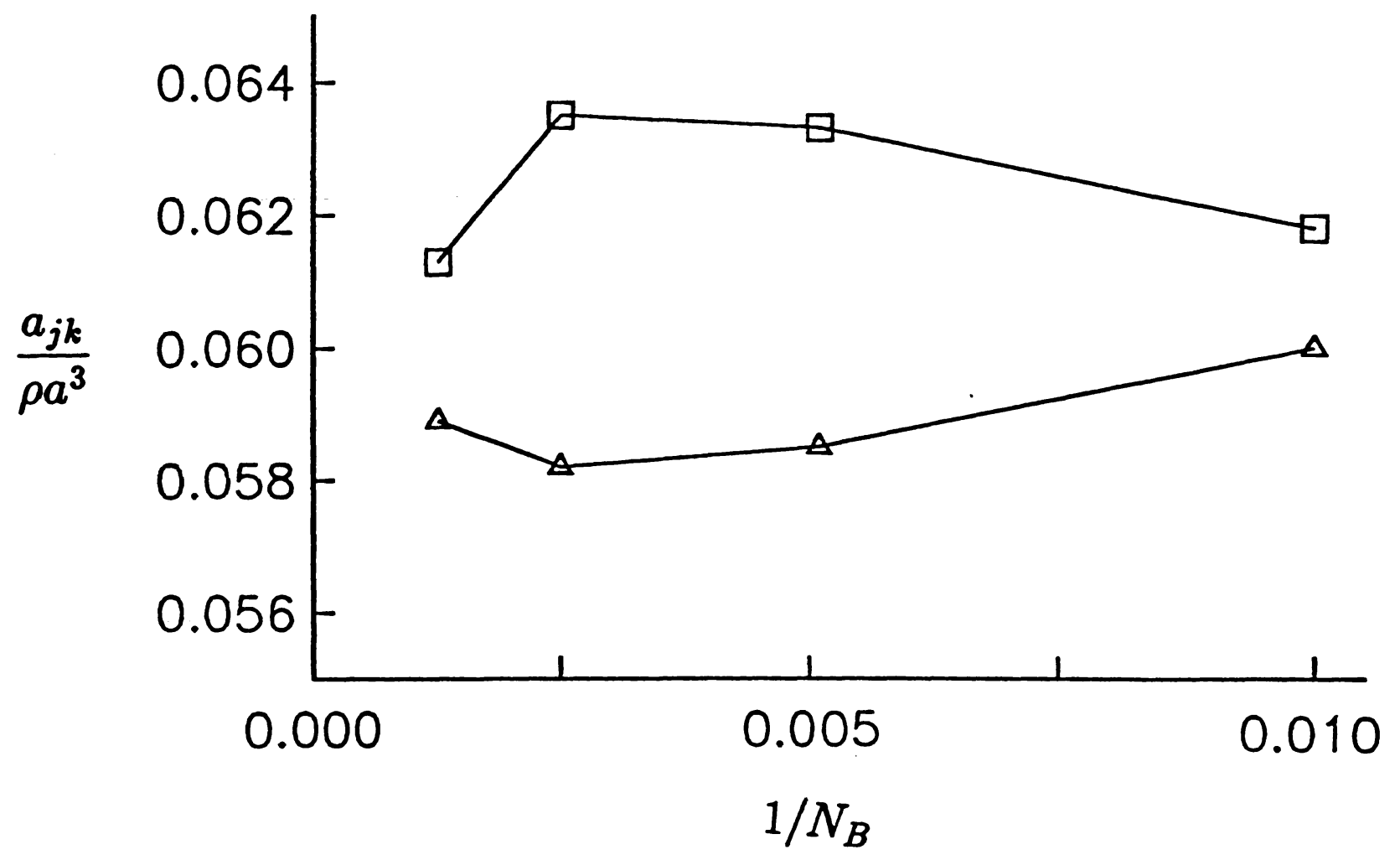

Figure 11.

$a_{13}(U)$ (squares) and $a_{31}(-U)$ (triangles) for a restrained half-immersed sphere vs. $1 / N_{B}$. $N_{F} \simeq 2.2 \times N_{B} . F r=0.04, h / a=1.2, \sigma^{2} a / g=0.8$. 
a)

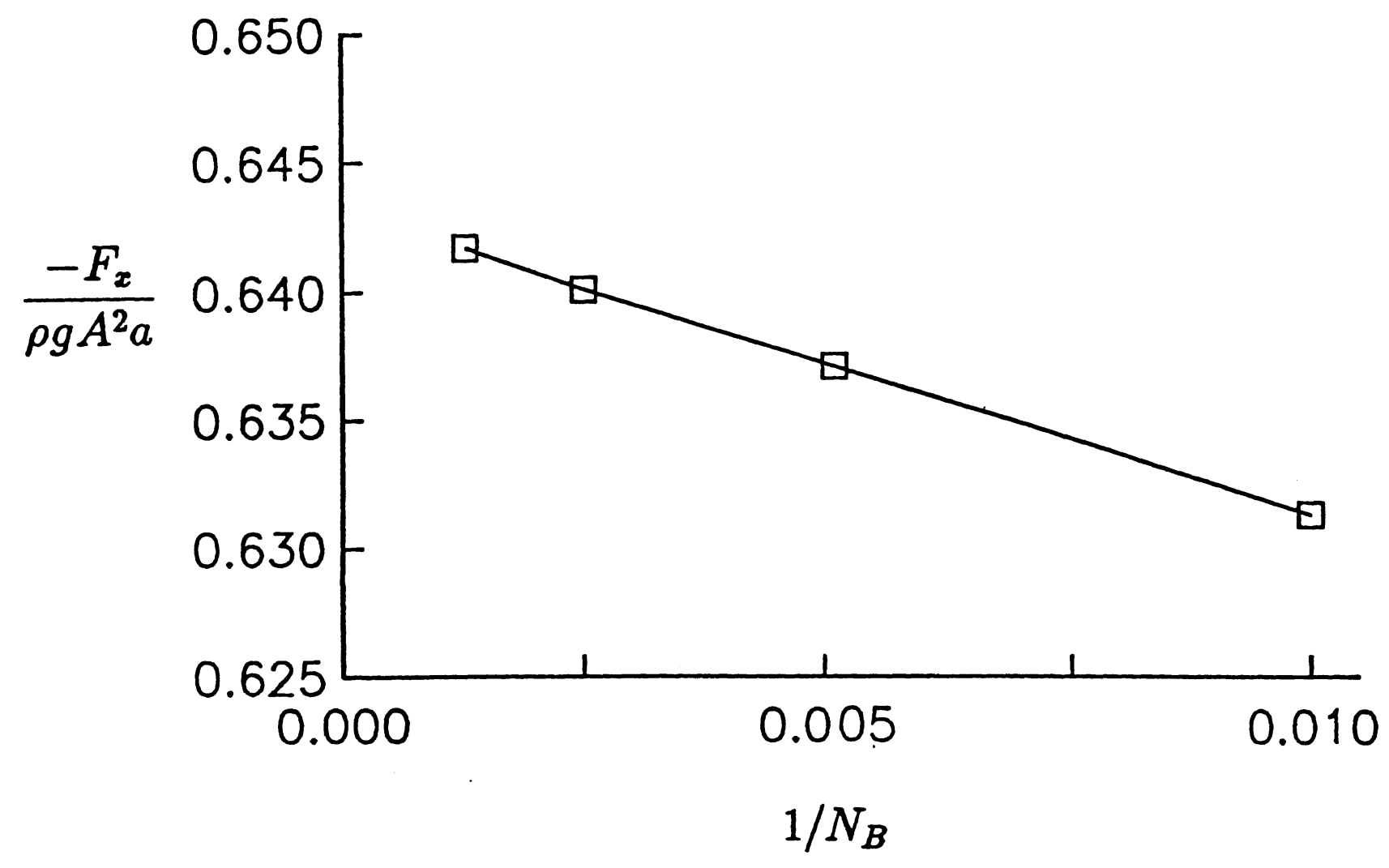

Figure 12

Mean drift force, $F_{x}$, and wave drift damping coefficient, $F_{x 1}$, for a restrained half-immersed sphere vs. $1 / N_{B} . N_{F} \simeq 2.2 \times N_{B} . h / a=1.2, K a=1.1391, \beta=\pi$. a) $F_{x}$ at $F r=0$. b) $F_{x}$ at $F r=0.04$. c) Wave drift damping coefficient $F_{x 1}$. 
b)

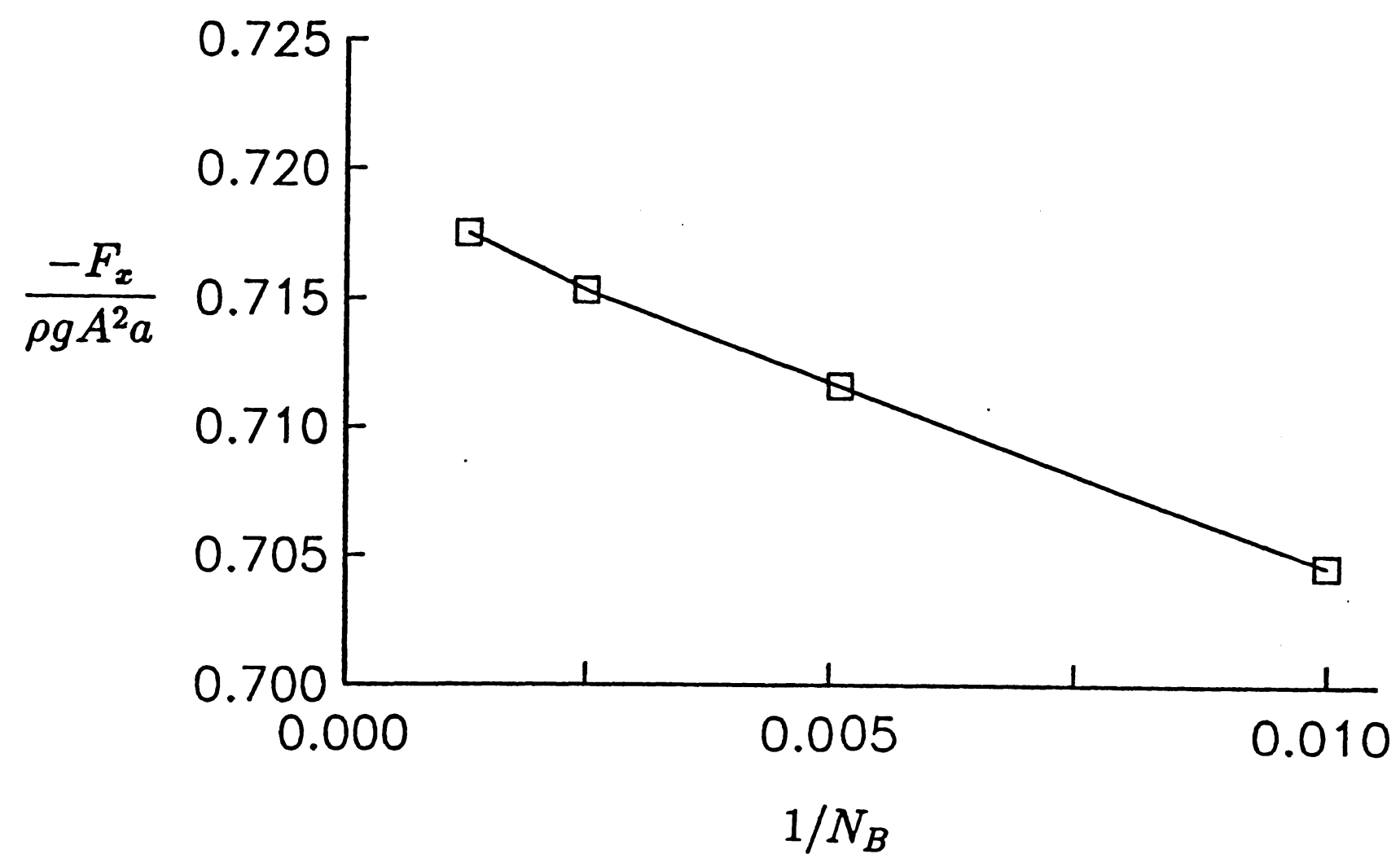

Figure 12

Mean drift force, $F_{x}$, and wave drift damping coefficient, $F_{x 1}$, for a restrained half-immersed sphere vs. $1 / N_{B} . N_{F} \simeq 2.2 \times N_{B} \cdot h / a=1.2, K a=1.1391, \beta=\pi$. a) $F_{x}$ at $F r=0$. b) $F_{x}$ at $F r=0.04$. c) Wave drift damping coefficient $F_{x 1}$. 
c)

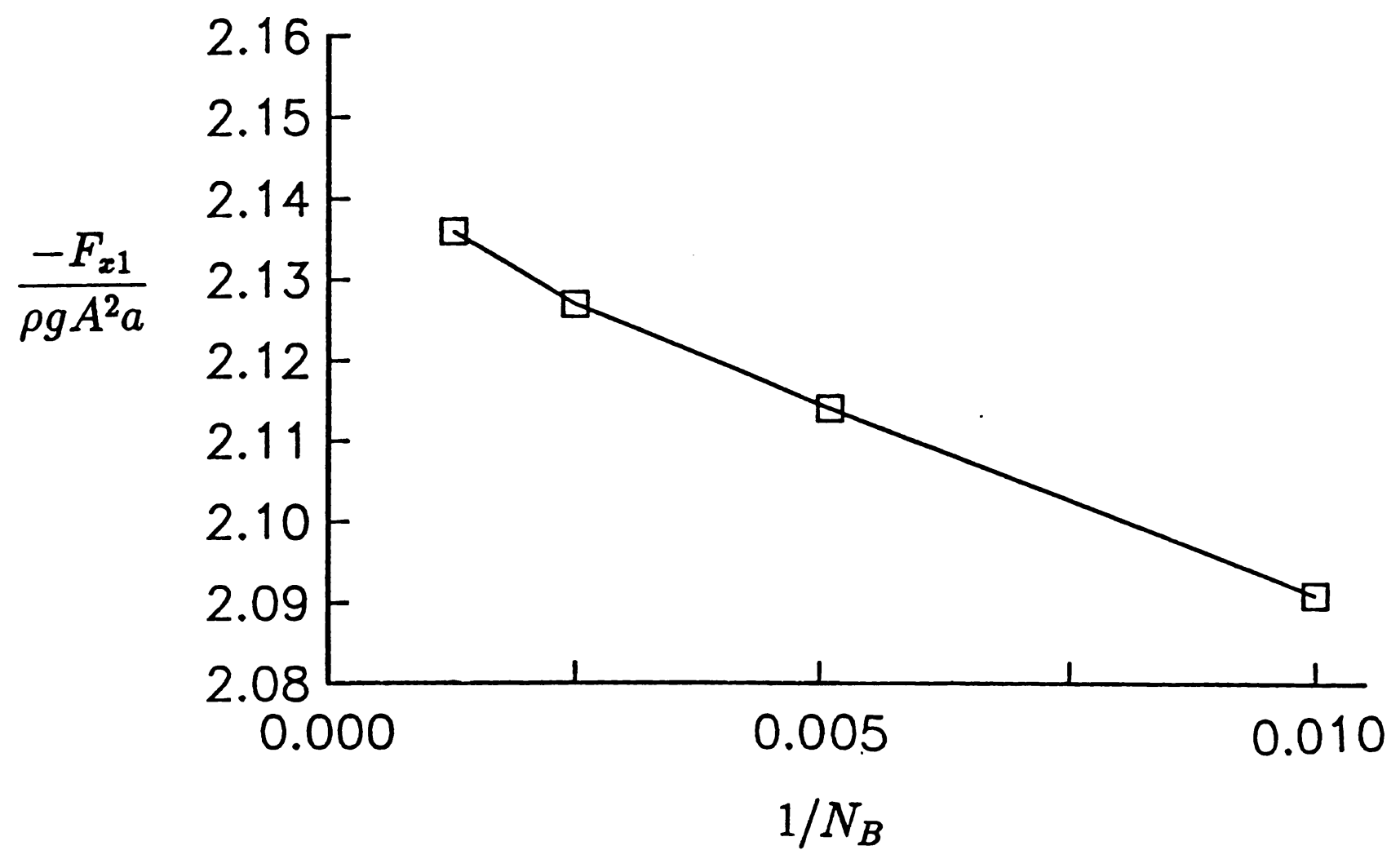

Figure 12

Mean drift force, $F_{x}$, and wave drift damping coefficient, $F_{x 1}$, for a restrained half-immersed sphere vs. $1 / N_{B} . N_{F} \simeq 2.2 \times N_{B} . h / a=1.2, K a=1.1391, \beta=\pi$. a) $F_{x}$ at $F r=0$. b) $F_{x}$ at $F r=0.04$. c) Wave drift damping coefficient $F_{x 1}$. 


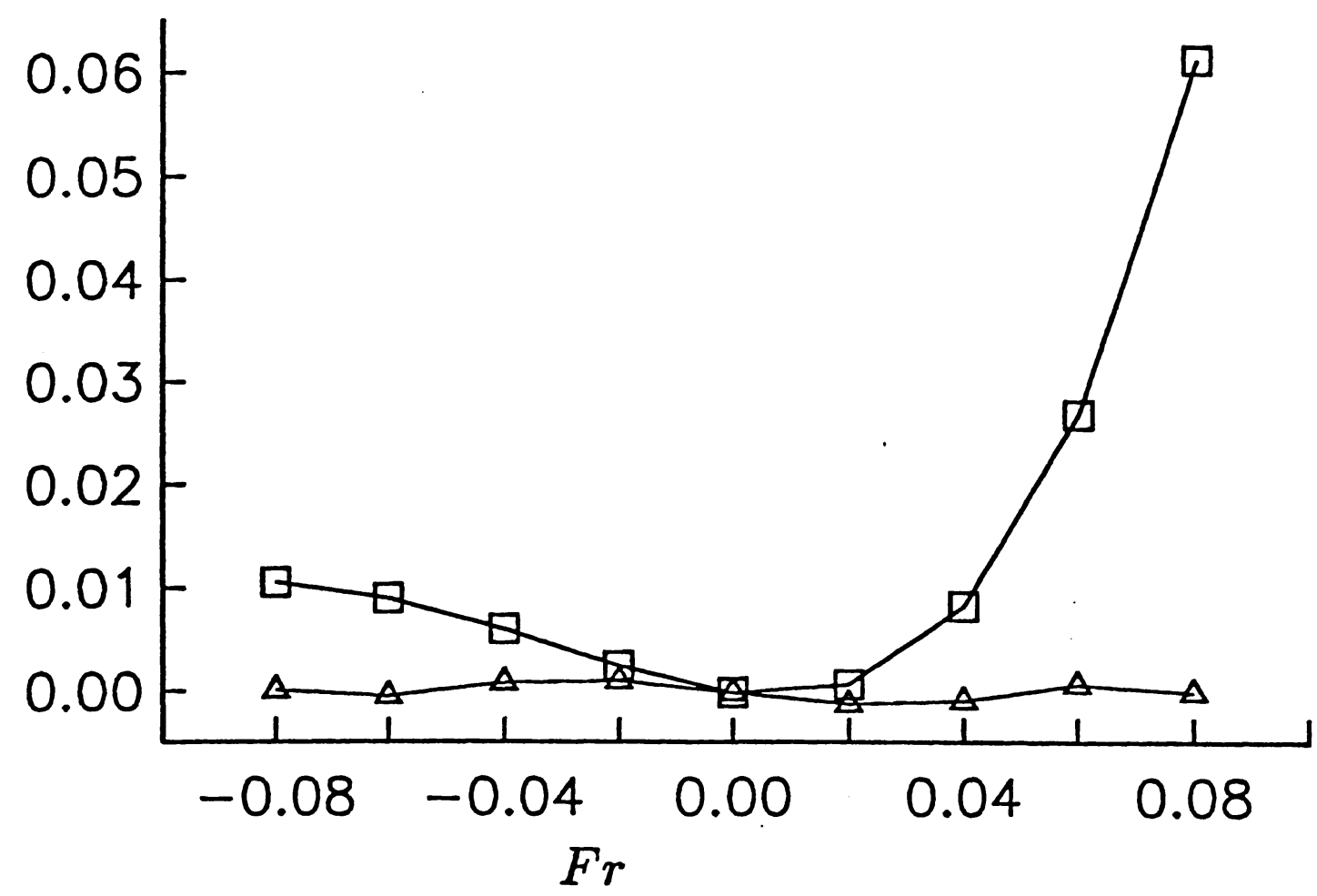

Figure 13

Energy flux at $S_{\infty}$ due to a restrained half-immersed sphere vs. Froude number. $W / 2 a E c_{g}$ (squares) and $W^{\prime} / 2 a E c_{g}$ (triangles). $h / a=1.2, K a=1.1391$ and $\beta=\pi$. Same discretization as in figure 2. 
